\title{
Topological Frequency Conversion in Strongly Driven Quantum Systems
}

\author{
Ivar Martin, ${ }^{1,2}$ Gil Refael, ${ }^{3,4}$ and Bertrand Halperin ${ }^{5}$ \\ ${ }^{1}$ Materials Science Division, Argonne National Laboratory, Argonne, Illinois 60439, USA \\ ${ }^{2}$ Kavli Institute for Theoretical Physics, University of California, Santa Barbara, California 93106, USA \\ ${ }^{3}$ Institute of Quantum Information and Matter and Department of Physics, \\ Pasadena, California 91125, USA \\ ${ }^{4}$ Walter Burke Institute of Theoretical Physics, California Institute of Technology, \\ Pasadena, California 91125, USA \\ ${ }^{5}$ Department of Physics, Harvard University, Cambridge, Massachusetts 02138, USA
}

(Received 25 January 2017; published 16 October 2017)

\begin{abstract}
When a physical system is subjected to a strong external multifrequency drive, its dynamics can be conveniently represented in the multidimensional Floquet lattice. The number of Floquet lattice dimensions equals the number of irrationally-related drive frequencies, and the evolution occurs in response to a built-in effective "electric" field, whose components are proportional to the corresponding drive frequencies. The mapping allows us to engineer and study temporal analogs of many real-space phenomena. Here, we focus on the specific example of a two-level system under a two-frequency drive that induces topologically nontrivial band structure in the 2D Floquet space. The observable consequence of such a construction is the quantized pumping of energy between the sources with frequencies $\omega_{1}$ and $\omega_{2}$. When the system is initialized into a Floquet band with the Chern number $C$, the pumping occurs at a rate $P_{12}=-P_{21}=(C / 2 \pi) \hbar \omega_{1} \omega_{2}$, an exact counterpart of the transverse current in a conventional topological insulator.

DOI: 10.1103/PhysRevX.7.041008

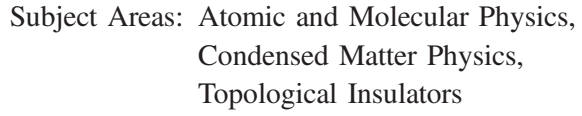

\section{INTRODUCTION}

A major goal of quantum condensed-matter physics is the control of many-body electronic and atomic states. A periodic drive is emerging as one of the most exciting means for achieving such control. Many proposals for phases that could be induced by periodic drive, so-called Floquet phases, have been made recently. References [1-5] demonstrate driving a band insulator into a topological phase using circularly polarized radiation or an alternating Zeeman field. References [6-9] explored topological invariants unique to periodically driven phases and predicted the Anderson-Floquet anomalous insulator, an unusual system with fully localized bulk but protected edge states. A general invariant for interacting systems was proposed in Refs. [10-13]. References [14-17] even showed that a driven disordered system in one dimension (1D) could spontaneously break the discrete time translation symmetry, forming the long-sought-after time crystal, while Refs. [18-20] showed that a periodic drive can delocalize a many-body localized system. This long list is a clear indication for the richness of Floquet engineering, with

Published by the American Physical Society under the terms of the Creative Commons Attribution 4.0 International license. Further distribution of this work must maintain attribution to the author(s) and the published article's title, journal citation, and DOI. some of the proposals already having attracted nascent experimental efforts [21-23].

A periodic drive alters the form of a quantum wave function. Each state becomes dressed by all possible harmonics of the drive frequency. The extra degrees of freedom associated with the amplitudes of the various drive harmonics effectively raise the dimensionality of the system and allow it to exhibit new phenomena. While this observation is at the basis of some of the work mentioned above, the role of this extra emergent dimension remains little utilized, with notable exceptions. This exact logic was used to make an array of optical oscillators into a 1D Thouless pump [24] and an array of two-dimensional (2D) oscillators into possessing Weyl points [25], and it even led to proposals for creating four-dimensional (4D) Hall phases using driven three-dimensional (3D) arrays of resonators [26].

Interestingly, there is another example where extra dimensions "magically" emerge: quasicrystals. Quasicrystals are aperiodic structures that could be understood as projections of higher-dimensional periodic crystals onto lower dimensions [27-29]. Most simply, a 1D quasicrystal can be constructed by superimposing two periodic but mutually incommensurate potentials.

Here, we show a surprising consequence of combining these two schemes for increasing the dimensionality of a system. Since the number of extra time dimensions is given by the number of applied drives with incommensurate 
(a)

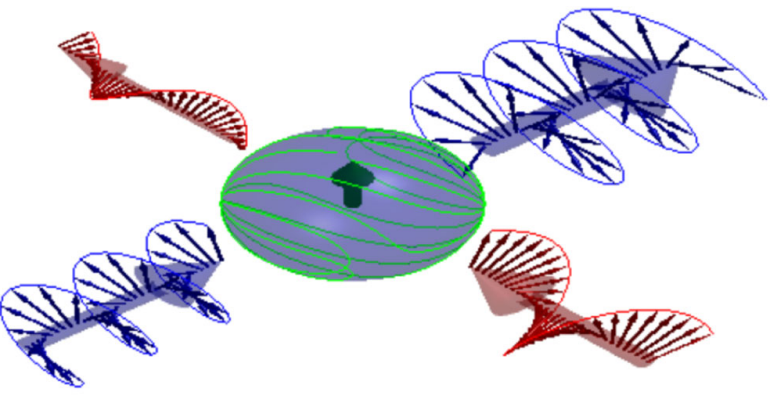

(b)

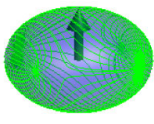

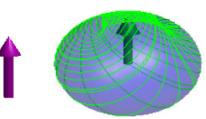

(c)

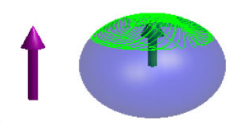

(d)

FIG. 1. We present a model that uses 2D topological insulator band structure as a guide to engineering the quantized energy pumping between different frequency modes. (a) A spin-1/2 particle driven by two sources with incommensurate frequencies. In the topological phase, the spin trajectory (green line) fully covers the Bloch sphere (blue). The intermode coupling induced by the spin dynamics pumps energy from one drive (red) to the other (blue) at a nearly quantized rate. The lower panel shows effective magnetic-field trajectories for (b) $m=0$ (gapless phase between two topological ones), (c) $m=1.8$ (just inside the topological phase), and (d) $m=2.2$ (just outside the topological phase). The mass $m$ is depicted as a purple arrow [static $B_{z}$ field; see Eq. (15) for the definition].

frequencies, one should be able to produce topological phenomena rooted solely in the time dimension. In particular, we demonstrate that subjecting a single spin- $1 / 2$ particle to two elliptically polarized periodic waves can realize the chiral Bernevig-Hughes-Zhang (BHZ) model [30], which usually resides in two spatial dimensions (see Fig. 1).

Just as a quantized Hall (spin or charge) conductance is the earmark of spatial topological phenomena, the signature of temporal topological phenomena arising from incommensurate drives is energy pumping. First, we show that by combining drives into a topological temporal texture, the system will pump energy between the driving fields, drawing energy from one and feeding it into the other. This general principle may be of practical importance and could be used, for instance, to convert photons between distinct photonic modes in optical cavities (in contrast to the perturbative nonlinearities that lead to the more conventional phenomena, such as frequency halving or doublinge.g., Ref. [31]). The pumping effect on its own will occur both in rational and irrational frequency combinations.

Second, the energy pumping rate between two incommensurate drives will be quantized. The energy pumping rate, averaged over time, and in the strong-drive limit, will be

$$
P=\omega_{1} \omega_{2} \frac{C}{2 \pi},
$$

with $\omega_{1}, \omega_{2}$ the two incommensurate drive frequencies, and $C$ the Chern number of the relevant topological band. Pure drives that are mutually rational, as we will show, may exhibit nonquantized pumping, even when the Chern number of the synthetic band is $C=0$. The topological nature of the driven system, however, will lend the pumping effect its robustness against disorder. As soon as temporal noise in the drives occurs (as long as it is not too strong to violate the "strong-drive" requirement), the average pumping rate would reduce to Eq. (D2), and no pumping will take place, on average, in a topologically trivial drive.

Our paper is organized as follows. After providing some background on Floquet theory and the physics of the Wannier-Stark ladder, we consider the general properties of a doubly driven system in Sec. III. Next, in Sec. IV, we introduce the BHZ path to temporal topological systems and explore the model's properties. In Sec. V, we explore the temporal BHZ model numerically and show that the topological regime is characterized by energy pumping. In Sec. VI, we explore the effects of temporal disorder and show that the topological drive lends the pumping effect robustness against disorder, for both mutually rational and incommensurate drives. In Sec. VII, we discuss the connection of our results with other systems and recent discoveries, and in Sec. VIII, we briefly discuss some other directions that can be pursued by means of multifrequency Floquet engineering.

\section{BACKGROUND}

\section{A. Floquet theory and the Floquet lattice}

The case of a simple periodic drive of arbitrary strength can be conveniently treated by Floquet theory [32], which is a time analog of Bloch theory for particles in spatially periodic potentials. In Bloch theory [33], particles carry quasimomentum. Analogously, in Floquet theory, when a system is driven by a single frequency $\omega$ (and its harmonics), the energy of eigenstates is replaced by quasienergy $E$. Instead of diagonalizing a Hamiltonian, the Floquet eigenstates (FE) diagonalize the single-period evolution operator,

$$
U_{T}=\mathcal{T} \exp \left(-i \int_{0}^{T} H(t) d t\right)
$$

where $H(t)$ is the time-dependent Hamiltonian, $\mathcal{T}$ is the time-ordering operator, and $T$ is the time period of the drive. Therefore, Floquet eigenstates have the form

$$
\Psi(t)=e^{-i E t} \Phi(t), \quad U_{T} \Phi(0)=e^{-i E T} \Phi(0) .
$$

Furthermore, the time-periodic part of the wave function can be expanded in the Fourier series, 


$$
\Phi(t)=\sum_{n} e^{-i n \omega t} \Phi_{n}
$$

and the index of the expansion harmonic $n$ can be interpreted as a position in the Floquet lattice. It has the physical meaning of the number of photons absorbed or emitted by the system. Substituting this form into the Schrödinger equation results in the following tight-binding eigenvalue problem:

$$
E \Phi_{n}=\sum_{m} h_{m-n} \Phi_{m}-\omega n \Phi_{n}
$$

with $h_{\ell}$ the $\ell$ th Fourier component of $H(t)$. Indeed, the count of absorbed photons emerges as an effective force field, and the tight-binding model resembles a WannierStark ladder [34] and, in the strong-drive limit, exhibits Bloch oscillations [35].

The standard-single-frequency-Floquet theorem can be extended to the case of multiple incommensurate frequencies. It has been utilized in the study of intense laser fields acting on atomic and molecular systems [36], as well as to simulate the Anderson localization in dimensions higher than one [37]. In both cases, the physical system subjected to the drive was zero dimensional. Clearly, the number of independent frequencies translates directly into the number of dimensions of the Floquet lattice.

\section{B. Wannier-Stark lattice and Berry curvature}

A peculiar feature of Floquet lattices generated by incommensurate frequency drives is that they always experience an effective uniform "electric field" applied in a noncrystallographic direction. This follows from Eq. (5), as soon as we add additional drives, as described in detail in the next section (Sec. III).

The projection of the field onto a particular lattice direction is proportional to the corresponding drive frequency

$$
\mathcal{H}_{\vec{\omega}}=\sum_{i=1}^{N} n_{i} \omega_{i}=\vec{n} \cdot \vec{\omega},
$$

where $\vec{n}$ is the integer vector of the drive harmonics, and $\vec{\omega}$ is a vector containing the angular frequencies of the drives. In analogy to the one-dimensional Wannier-Stark ladders, if the potential-energy drop over a lattice constant exceeds the hopping, then the band-structure effects are lost (this corresponds to the weak-drive regime). On the other hand, for the strong-drive regime, the effective electric field (i.e., frequency) plays the role of a perturbation, and the band description provides a convenient framework to study deviations from adiabaticity.

What are the physical implications of the topologically nontrivial Floquet band structure in the strong-drive case? In conventional materials, bulk topological invariants, and
Chern numbers in particular, lead to the appearance of gapless edge modes [38]. In the case of the Floquet lattice, for a classical coherent drive, the lattice does not have a boundary (producing such a boundary requires specifically tailored history dependence and is beyond the scope of this work). Nevertheless, the pseudoelectric field can induce chiral propagation, mimicking the edge physics in a finite crystal. Analogously, we can think of the effective force as inducing an anomalous velocity, which in two and three dimensions is

$$
\vec{v}=\vec{\omega} \times \vec{\Omega}_{\vec{q}}
$$

where $\vec{\Omega}_{\vec{q}}$ is the Berry curvature in the Floquet zone, defined in Eq. (25).

The chiral propagation on the Floquet lattice corresponds to the energy transfer between the individual drives. In the case of the two-frequency drive of a two-level system, as we will show, the pumping power, averaged over long times, is proportional to the product of frequencies and the Chern number of the band into which the system is initialized.

Contrary to what would be expected in the standard (perturbative) $n$-wave mixing, in the strong-drive regime, any frequency can be converted into any other, whether they are rationally or irrationally related. The topological quantization of the energy pumping in the case of two rationally related drive frequencies, with no disorder, will be absent. The nonquantized effect, however, is not robust against temporal disorder in the drive. When such disorder is present, as we demonstrate in Sec. VI, even a rational frequency ratio will result in an averaged energy pumping rate that is proportional to the Chern number of the band.

\section{FLOQUET REPRESENTATION FOR MULTIPLE-DRIVE FREQUENCIES}

The basis for our work is a mapping between a system of $d$ spatial dimensions subjected to $N$ mutually irrational drives and a $d+N$-dimensional system. In rough terms, the extra $n$ dimensions in the multidrive Floquet problem emerge when we consider the number of energy quanta absorbed from each drive. As we show below, the numbers of photons absorbed from each drive make up the coordinates in an $n$-dimensional lattice. The energy associated with each photon, however, gives rise to an effective force in this lattice description since the Hamiltonian will contain diagonal terms $\sum_{i} n_{i} \omega_{i}$, with $n_{i}$ the number of absorbed photons from the drive with angular frequency $\omega_{i}$. Below, we make this analogy more precise.

Consider a system with basis states $|\alpha\rangle$, with $\alpha=1,2, \ldots$ (for instance, spin states-though they could also be position states in real space) subject to a Hamiltonian

$$
\mathcal{H}=\sum_{\alpha, \beta} H^{\alpha \beta}\left(\varphi_{1}, \varphi_{2}, \ldots\right)|\alpha\rangle\langle\beta|,
$$


such that each element $H^{\alpha \beta}\left(\varphi_{1}, \varphi_{2}, \ldots\right)$ is periodic for $\varphi_{i} \rightarrow \varphi_{i}+2 \pi$. We assume linear time dependence for the $\varphi_{i}$ 's,

$$
\varphi_{i}(t)=\omega_{i} t,
$$

with the $\omega_{i}$ 's being mutually incommensurate. The Schrödinger equation, written in terms of the components $\psi^{\alpha}$ of the wave function $|\psi\rangle=\sum_{\alpha} \psi^{\alpha}|\alpha\rangle$, is

$$
i \partial_{t} \psi^{\alpha}(t)=H^{\alpha \beta}[\vec{\varphi}(t)] \psi^{\beta}(t) .
$$

Equation (10) represents a system under multitone drive. It can be analyzed in the spirit of Floquet theorem for a single drive. Following Floquet's construction, we write the wave function as

$$
|\psi(t)\rangle=e^{-i E t} \sum_{\alpha, n_{1}, n_{2}, \ldots} \phi_{\vec{n}}^{\alpha} e^{-i \vec{n} \cdot \vec{\omega} t}|\alpha\rangle .
$$

Above, we introduced the vector notation $\vec{n} \cdot \vec{\omega}=\sum_{i} n_{i} \omega_{i}$. The Hamiltonian can also be expanded in terms of its Fourier components,

$$
\mathcal{H}^{\alpha \beta}(\vec{\varphi})=\sum_{\vec{p}} h_{\vec{p}}^{\alpha \beta} e^{-i \vec{p} \cdot \vec{\varphi}} .
$$

Next, we combine Eqs. (10)-(12) and obtain a tightbinding eigenproblem [39]

$$
(E+\vec{n} \cdot \vec{\omega}) \phi_{\vec{n}}^{\alpha}=\sum_{\vec{p}} h_{\vec{p}}^{\alpha \beta} \phi_{\vec{n}-\vec{p}}^{\beta} .
$$

Once more, we made use of the vector notation for the set of integers $p_{i}$.

Equation (13) describes a hopping problem on what we will refer to as a Floquet lattice (see Fig. 2). It has as many dimensions as there are independent drive terms. In addition, it has a tilt, with the potential $U(\vec{n})=-\vec{n} \cdot \vec{\omega}$. This makes intuitive the interpretation of the Floquet lattice: $n_{i}$ is the number of photons absorbed by the system from drive $i$. Accordingly, if the energy spectrum of the system in the absence of a drive is bounded, the wave function $\phi_{\vec{n}}^{\alpha}$ will be exponentially confined to a strip normal to the direction $\vec{\omega}$, that is, in the direction $\left(-\omega_{2}, \omega_{1}\right)$. Along the strips, the wave functions should be nondivergent, which fully specifies the eigenvalue-eigenvector problem (13). Naively, the number of eigenstates is equal to the lattice size times the dimension of the local Hilbert space. However, it is easy to see that the number of independent Floquet eigenstates is the same as for the undriven system. Indeed, Eq. (13) has a symmetry: If $\phi_{\vec{n}}^{(1) \alpha}$ is a solution with quasienergy $E^{(1)}$, then $\phi_{\vec{n}}^{(2) \alpha}=\phi_{\vec{n}-\vec{m}}^{(1) \alpha}$ is also a solution with $E^{(2)}=E^{(1)}-\vec{m} \cdot \vec{\omega}$ for any integer vector $\vec{m}$. Indeed, both correspond to the identical solution of the time-dependent Schrödinger equation (10). This can be verified explicitly,

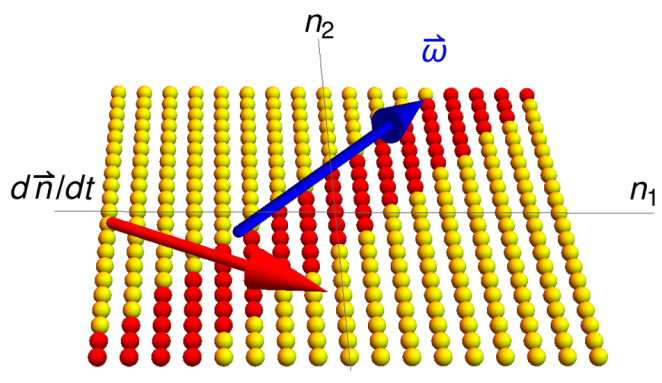

FIG. 2. The Floquet harmonics in a two-drive system give rise to a two-dimensional space. Each spot represents the $n_{1} \omega_{1}+$ $n_{2} \omega_{2}=\vec{n} \cdot \vec{\omega}$ harmonic. If the frequencies are incommensurate, then the space is infinite. If the ratio $\omega_{1} / \omega_{2}$ is rational, however, the infinite lattice contracts to a cylinder (strip marked in red, with periodic boundary conditions across the strip). The periodic drives appear to exert a force in the $\vec{n}$ space along the $\vec{\omega}$ direction. The motion that arises because of Berry curvature is normal to the force and is indicated by the $d \vec{n} / d t$ arrow.

$$
\begin{aligned}
\psi^{(2) \alpha}(t) & =\sum_{\vec{n}} e^{-i E^{(2)} t-i \vec{n} \cdot \vec{\omega} t} \boldsymbol{\phi}_{\vec{n}}^{(2) \alpha} \\
& =\sum_{\vec{n}} e^{-i E^{(2)} t-i \vec{n} \cdot \vec{\omega} t} \boldsymbol{\phi}_{\vec{n}-\vec{m}}^{(1) \alpha} \\
& =\sum_{\vec{n}} e^{-i\left(E^{(2)}+\vec{m} \cdot \vec{\omega}\right) t-i \vec{n} \cdot \vec{\omega} t} \boldsymbol{\phi}_{\vec{n}}^{(1) \alpha} \\
& =\psi^{(1) \alpha}(t) .
\end{aligned}
$$

Any initial wave function at $t=0$ can be expanded in terms of the unique Floquet eigenstates.

If not for the linear on-site potential on the lhs of Eq. (13), the problem would be a translationally invariant tight-binding model in the Floquet space and could be trivially solved by a Fourier transform. The Floquet wave functions would be $\phi_{\vec{n}}^{\alpha}(\vec{\varphi})=\tilde{\phi}^{\alpha}(\vec{\varphi}) e^{i \vec{\varphi} \cdot \vec{n}}$. Remarkably, the energy eigenvalues in the absence of the drive $\epsilon^{(i)}(\vec{\varphi})$ are the eigenvalues of $\mathcal{H}(\vec{\varphi})$ from Eq. (8). Thus, the angles $\vec{\varphi}$ play the role of momentum for the driven system. In analogy to the Bloch and Brillouin cases, we refer to the region $0<\varphi_{i}<2 \pi$ as the Floquet zone.

With tilted potential turned on, the Floquet lattice eigenvalue problem becomes equivalent to the Stark ladder in 2D [40]. The tilt produces a change in the momentum angles as

$$
\vec{\varphi}=\vec{\varphi}_{0}+\vec{\omega} t
$$

To find the effects of the tilt on the wave functions, first consider the situation where the driving is strong, which is equivalent to the level spacings of $\epsilon^{(i)}(\vec{\varphi})$ being much greater than the largest angular frequency. In this case, we can describe the system using a semiclassical approach following the motion of a particle with momentum $\vec{\varphi}$ in the $\vec{n}$ lattice space. In this case, if the band structure is topologically nontrivial, we can obtain chiral "edge" modes 
that drift along the equipotential lines in $\vec{n}$. This will be central to our work.

In the weak driving case, we obtain an effective 1D hopping model that describes quasicrystal with variable onsite potential (and variable hopping, if desired). This regime is not central to the main subject of the paper, but interested readers may refer to Appendix A.

Let us next consider how the construction above changes when the frequencies have a rational ratio. Consider a twodrive system, with $\omega_{1} p=\omega_{2} q$, with $p$ and $q$ mutually prime. First, the mapping (shown above) of the Floquet problem to a $2 \mathrm{D}$ lattice representing the different frequency components of the generalized Floquet wave function becomes redundant. The rational ratio can be taken into account by identifying the $\left(n_{1}, n_{2}\right)$ lattice point with $\left(n_{1}+m p, n_{2}-m q\right)$ for any integer $m$. This compactifies the 2D lattice into a strip with periodic boundary conditions, and the vector $(p,-q)$ connects equivalent points across the strip's boundary. So, essentially, the problem is thereby reduced to that of a cylinder of circumference $\sqrt{p^{2}+q^{2}}$, made of a square lattice (Fig. 2; for detailed treatment, see Appendix B). As we show in the following, for large $\sqrt{p^{2}+q^{2}}$, there is no significant difference between the rational and irrational cases as far the energy pumping efficiency is concerned. The topologically quantized value is obtained as $\sqrt{p^{2}+q^{2}} \rightarrow \infty$.

A related observation, interesting from a mathematical perspective, is that it is possible to define Floquet eigenstates for quasiperiodic drive, by means of a limiting procedure. Specifically, we can approximate any irrational frequency ratio as a limit of a ratio of two integers both tending to infinity, $\omega_{1} / \omega_{2}=\lim _{i \rightarrow \infty}\left(q_{i} / p_{i}\right)$. Solving a sequence of Floquet eigenstate problems for progressively smaller $\omega_{i}=\left(\omega_{1} / q_{i}\right)=\left(\omega_{2} / p_{i}\right)$, we find that the eigenstates indeed converge to a limiting state that one can define as the quasiperiodic Floquet eigenstate. The demonstration of this result is given in Appendix C.

\section{TEMPORAL TOPOLOGICAL SYSTEMS}

The analogy between multiple incommensurate drives and multidimensional lattice quantum dynamics allows us to constructs zero-dimensional topological systems. The topological effects will arise from the temporal structure of the wave functions of the driven system. Below, we construct such a system, which consists of a single spin$1 / 2$ particle, driven by two incommensurate periodic drives. We first define the model and then explore its topological properties. In particular, we consider the semiclassical motion of the system on the Floquet lattice. We concentrate on the associated $\vec{\varphi}$ momentum space, especially when there is Berry curvature associated with the Floquet-lattice momentum states $\psi^{\alpha}(\vec{\varphi})$. Figure 3 depicts all elements relevant to the discussion and maps out the

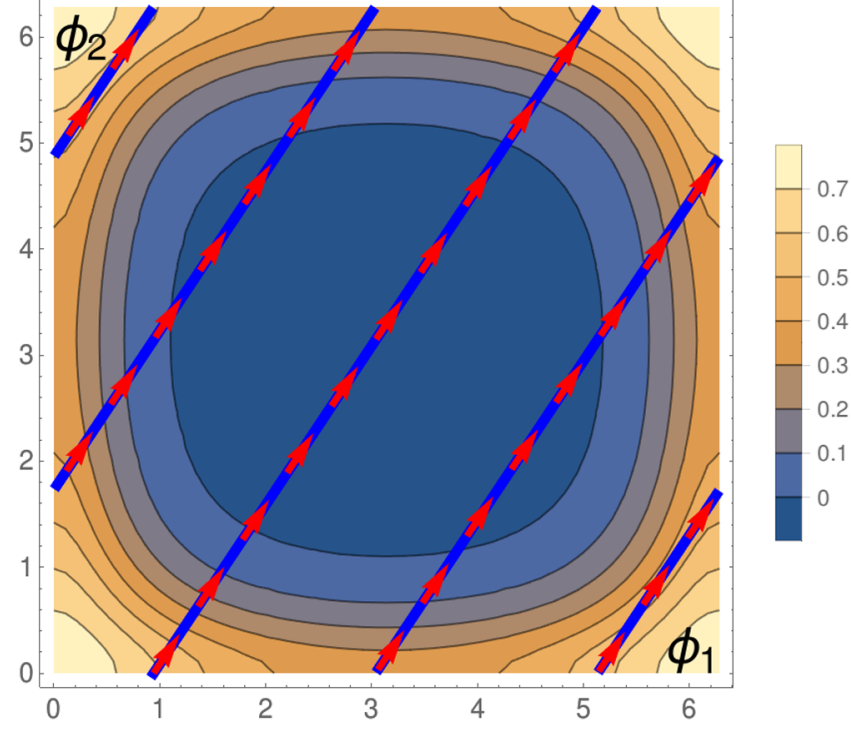

FIG. 3. The Floquet zone for two drives. In analogy to a 2D band structure, we draw the Berry curvature (color plot with side legend) vs the two offset phases $\varphi_{1}$ and $\varphi_{2}$. The periodic drives are akin to a motion along this Floquet zone with $\vec{\varphi}=\vec{\varphi}_{0}+\vec{\omega} t$. In the case of a rational frequency ratio, the system will explore a closed periodic path through the Floquet zone. Drawn here is an example of $3 \omega_{1}=2 \omega_{2}$. The pumping effect will be roughly the integral of the Berry curvature along the path times $\omega_{1} \omega_{2}$ [Eq. (22)].

motion of the system in the momentum $\vec{\varphi}$ space, as well as the Berry curvature of the model defined below.

\section{A. BHZ path to temporal topological physics}

Let us choose $h_{p q}^{\alpha \beta}$ in Eq. (13) so that the translationally invariant tight-binding band structure is topologically nontrivial. One of the simplest band structures of this type is half of the BHZ model [30], with the Hamiltonian

$$
\begin{aligned}
\mathcal{H}= & v_{x} \sin \left(k_{1}\right) \sigma_{x}+v_{y} \sin \left(k_{2}\right) \sigma_{y} \\
& +\left[m-b_{x} \cos \left(k_{1}\right)-b_{y} \cos \left(k_{2}\right)\right] \sigma_{z} .
\end{aligned}
$$

The model yields a quantum Hall insulator (band Chern numbers \pm 1$)$ for $-\left|b_{1}\right|-\left|b_{2}\right|<m<-|| b_{1}|-| b_{2}||$ and ||$b_{1}|-| b_{2}||<m<\left|b_{1}\right|+\left|b_{2}\right|$. The corresponding Floquet Hamiltonian is obtained by replacing $k_{1} \rightarrow \omega_{1} t+\varphi_{1}$ and $k_{2} \rightarrow \omega_{2} t+\varphi_{2}$,

$$
\begin{aligned}
\mathcal{H}= & v_{1} \sin \left(\omega_{1} t+\varphi_{1}\right) \sigma_{x}+v_{2} \sin \left(\omega_{2} t+\varphi_{2}\right) \sigma_{y} \\
& +\left[m-b_{1} \cos \left(\omega_{1} t+\varphi_{1}\right)-b_{2} \cos \left(\omega_{2} t+\varphi_{2}\right)\right] \sigma_{z} .
\end{aligned}
$$

The interpretation of this Hamiltonian harks back to the discussion of the emerging Floquet lattice of Sec. III. The operator $e^{-i \omega_{i} t-i \varphi_{i}}$ absorbs a photon from drive $i$, thus realizing a hop on the Floquet lattice in the direction $i$. In order to be able to follow the time evolution on the Floquet 
lattice, let us explicitly extend the Hilbert space to the direct product of the spin and lattice spaces, with the wave function $|\psi\rangle=\sum_{\alpha \vec{n}} \psi_{\vec{n}}^{\alpha}|\vec{n}\rangle|\alpha\rangle$ [compare with Eq. (11)]. In this representation, $e^{ \pm i \omega_{i} t} \rightarrow\left|n_{i} \mp 1\right\rangle\left\langle n_{i}\right|$. We obtain a tight-binding Schrödinger equation for the wave-function amplitude on the Floquet lattice,

$$
\begin{aligned}
i \partial_{t} \psi_{n_{1}, n_{2}}= & \left.\mathcal{H} \psi\right|_{n_{1}, n_{2}}=\frac{1}{2}\left(i v_{1} \sigma_{x}-b_{1} \sigma_{z}\right) e^{i \varphi_{1}} \psi_{n_{1}-1, n_{2}} \\
& +\frac{1}{2}\left(-i v_{1} \sigma_{x}-b_{1} \sigma_{z}\right) e^{-i \varphi_{1}} \psi_{n_{1}+1, n_{2}} \\
& +\frac{1}{2}\left(i v_{2} \sigma_{y}-b_{2} \sigma_{z}\right) e^{i \varphi_{2}} \psi_{n_{1}, n_{2}-1} \\
& +\frac{1}{2}\left(-i v_{2} \sigma_{y}-b_{2} \sigma_{z}\right) e^{-i \varphi_{2}} \psi_{n_{1}, n_{2}+1} \\
& +\left(m \sigma_{z}-n_{1} \omega_{1}-n_{2} \omega_{2}\right) \psi_{n_{1}, n_{2}} .
\end{aligned}
$$

We could even go further and Fourier transform the hopping part of the Hamiltonian to obtain its "momentum representation" on the tight-binding Floquet lattice:

$$
\begin{aligned}
\mathcal{H}_{\vec{q}}= & v_{1} \sigma_{x} \sin \left(q_{1}+\varphi_{1}\right)+v_{2} \sigma_{y} \sin \left(q_{2}+\varphi_{2}\right) \\
& +\left[m-b_{1} \cos \left(q_{1}+\varphi_{1}\right)-b_{2} \cos \left(q_{2}+\varphi_{2}\right)\right] \sigma_{z}, \\
\mathcal{H}_{\vec{n}}= & -\vec{n} \cdot \vec{\omega}, \\
\mathcal{H}= & \sum_{\vec{q}} \mathcal{H}_{\vec{q}} \hat{n}_{\vec{q}}+\sum_{\vec{n}} \mathcal{H}_{\vec{n}} \hat{n}_{n},
\end{aligned}
$$

where $\hat{n}_{\vec{n}}$ is the occupation of site $\vec{n}=\left(n_{1}, n_{2}\right)$ and similarly $\hat{n}_{\vec{q}}$ is the occupation of the momentum state $\left(q_{1}, q_{2}\right)=\vec{q}$. The Hamiltonian written in the form Eq. (18) mixes the real (frequency) and momentum (phase or time) space representations. The diagonal part $\mathcal{H}_{\vec{n}}=\vec{n} \cdot \vec{\omega}$ has the role of a force, which pushes the momenta $\vec{q}$ in the $\vec{\omega}$ direction. This naturally leads to the momentum evolution

$$
\vec{q}=\vec{\omega} t .
$$

\section{B. Photon absorption rates}

What would be the manifestation of a topological band structure in the Floquet lattice? When the Berry curvature is present, the system will respond to the force implied by $\mathcal{H}_{\vec{n}}=-\vec{n} \cdot \vec{\omega}$ by moving normal to the force in a preferred direction determined by the band into which the system was initiated. From the practical standpoint, the "movement" of the particle within a chiral band corresponds to a process where photons of one frequency are absorbed and those of the other are emitted, in the proportions that approximately [up to uncertainty of the scale $\|h(t)\|$ ] conserve energy. In other words, the rates of work performed by "sources" with frequencies $\omega_{i}$ should add up to approximately zero on average, but each one can be substantial.

Let us derive expressions for the photon emission and absorption. From Eq. (18), we readily notice that the offset phases $\varphi_{i}$ act as a lattice vector potential for the photon flow. Therefore, the derivative

$$
\hat{j}_{i}=\frac{\partial \mathcal{H}}{\partial \varphi_{i}}=\frac{\partial n_{i}}{\partial t}
$$

is the current, or velocity operator, in the Floquet lattice. It is the rate of absorption or emission of photons with frequency $\omega_{i}$. The rate of energy absorption (power) is then

$$
\frac{\partial\left\langle E_{i}\right\rangle}{\partial t}=\omega_{i}\left\langle\hat{j}_{i}\right\rangle=\omega_{i} \frac{\partial\left\langle\hat{n}_{i}\right\rangle}{\partial t} .
$$

This intuitive result can also be obtained in another way. Consider a generic double-drive Hamiltonian as $\mathcal{H}=\vec{h}_{1}\left(\omega_{1} t+\varphi_{1}\right) \cdot \vec{\sigma}+\vec{h}_{2}\left(\omega_{2} t+\varphi_{2}\right) \cdot \vec{\sigma}$. The time derivative of the total energy is $d E / d t=d\langle H\rangle / d t=$ $i\langle[H, H]\rangle+\langle(\partial H / \partial t)\rangle=\left\{\left[\partial \vec{h}_{1}\left(\omega_{1} t+\varphi_{1}\right)\right] / \partial t\right\} \cdot\langle\vec{\sigma}\rangle+$ $\left\{\left[\partial \vec{h}_{2}\left(\omega_{2} t+\varphi_{2}\right)\right] / \partial t\right\} \cdot\langle\vec{\sigma}\rangle$. Therefore, change of energy due to a given source is

$$
\frac{\partial\left\langle E_{i}\right\rangle}{\partial t}=\omega_{i}\left\langle\frac{\partial \mathcal{H}}{\partial \phi_{i}}\right\rangle=\frac{\partial \vec{h}_{i}}{\partial t} \cdot\langle\vec{\sigma}\rangle
$$

Note that the same formalism can be applied not only to determine the work performed by a given frequency drive but also to separate the energy flows between different polarizations of the same frequency drive.

\section{Semiclassical equations of motion and pumping power}

The evolution of position on the Floquet lattice in the limit of a small applied "electric" field (that is, small frequency) can be determined from the semiclassical equations of motion. Within a particular band of $\mathcal{H}_{\vec{q}}$, they are [41]

$$
\begin{aligned}
& \dot{\vec{n}}=\nabla_{\vec{q}} \epsilon_{\vec{q}}-\nabla_{n} \mathcal{H}_{\vec{n}} \times \Omega_{\vec{q}}, \\
& \dot{\vec{q}}=-\nabla_{n} \mathcal{H}_{\vec{n}}=\vec{\omega} .
\end{aligned}
$$

In the Floquet problem, there is a constant "force" $\vec{\omega}$, and we take

$$
\vec{q}(t)=\vec{\omega} t,
$$

which, after substitution into Eq. (18), as expected, yields the original problem, Eq. (16). The key to our problem is the anomalous velocity, related to the Berry curvature (in $2 \mathrm{D}$ and $3 \mathrm{D})$

$$
\Omega_{\vec{q}}=\hat{z} i \frac{1}{2} \operatorname{tr}\left(P_{\vec{q}}\left[\frac{\partial P_{\vec{q}}}{\partial q_{1}}, \frac{\partial P_{\vec{q}}}{\partial q_{2}}\right]\right)
$$


with $P_{\vec{q}}$ a projector onto a particular band of $\mathcal{H}_{\vec{q}}$ [e.g., for the lower band, $P_{\vec{q}}=\left(1-\mathcal{H}_{\vec{q}} / \epsilon_{\vec{q}}\right) / 2$ ].

On average, only the Berry curvature pumps. The energy that goes between the two drives is then

$$
\frac{1}{2} \frac{\partial\left(E_{1}-E_{2}\right)}{\partial t}=\frac{1}{2}\left(\omega_{1},-\omega_{2}\right) \cdot\left(\vec{\omega} \times \Omega_{\vec{q}}\right)=\left|\Omega_{\vec{q}}\right| \omega_{1} \omega_{2} .
$$

\section{Quantum Hall analogy}

The above result can also be obtained by directly exploiting the analogy with the Hall response in the topological insulators. Namely, we use the known expression for the Hall current in order to determine the average drift velocity of an individual particle. We take unit cell size 1 and electric field $\overrightarrow{\mathcal{E}}=\left(\omega_{1}, \omega_{2}\right)$. The field points at an angle $\alpha=\arctan \omega_{2} / \omega_{1}$ to axis 1 . For one fully occupied band with Hall conductivity $\sigma_{x y}$, the application of the electric field leads to the transverse current density

$$
j_{\perp}=\sigma_{x y} \mathcal{E}
$$

which flows in the direction $\alpha+\pi / 2$. This current is a product of particle density and velocity, and since the size of a unit cell is 1 and the band is fully occupied, the density is 1 . Thus, the drift velocity is $v=\sigma_{x y} \sqrt{\omega_{1}^{2}+\omega_{2}^{2}}$. Its projection onto axis 1 is $v_{1}=v \sin \alpha=\sigma_{x y} \omega_{2}$; therefore, the rate of energy change along axis 1 , which is the same as the power exerted by mode 1 , is

$$
\frac{\partial E_{1}}{\partial t}=\sigma_{x y} \omega_{1} \omega_{2}=-\frac{\partial E_{2}}{\partial t},
$$

equivalent to Eq. (26). There is also an appealing connection to quantum pumping, described in Appendix D.

\section{E. Quantization and the role of incommensurability}

If over time the entire Floquet zone is probed by the spin, we obtain a topologically quantized pumping, as in Eq. (D2), since

$$
\overline{\Omega_{\vec{q}}}=\int \frac{d^{2} q}{(2 \pi)^{2}} \omega_{\vec{q}}=\frac{C}{2 \pi},
$$

with $C$ the Chern number of the band. For this to happen, the frequency ratio $\omega_{1} / \omega_{2}$ must be incommensurate. It is important to note that the quantization will emerge once the system has enough time to sample the Floquet zone.

If, on the other hand, the frequency ratio is a rational number, then, over time, the path in the Floquet zone $\vec{\omega} t$ $\bmod 2 \pi$ will repeat itself, and only part of the Berry curvature will be sampled. Therefore, for rational $\omega_{1} / \omega_{2}$, the pumping power is generally not quantized; however, the average over the phases $\varphi_{1}$ and $\varphi_{2}$ (which can be interpreted as initial conditions) is quantized.
Interestingly, the lack of quantization of the rationalfrequency pumping effect renders it susceptible to temporal disorder effects. Any temporal disorder effectively averages over the phase differences between the two drives and also forces an exploration of the entire two-parameter BrillouinFloquet zone. The pumping, on average, will again be quantized and proportional to the Chern number of the synthetic 2D space (see Sec. VI).

\section{RESULTS OF NUMERICAL SIMULATIONS}

Let us now explore the pumping effects described above numerically. We find that, indeed, in the topological parameter range, as long as the gap in the corresponding band Hamiltonian exceeds the drive frequencies (i.e., for a sufficiently strong drive), energy flows between the two drives at a nearly quantized rate. A necessary condition for strong pumping is the high "fidelity"-a large projection of the spin onto the direction of the instantaneous "magnetic field" (equivalent to the ability of the spin to stay in one topological band). We consider these for both incommensurate and commensurate drive frequencies. Interestingly, depending on the initial conditions, the pumping effect for the rational frequency ratio can exceed its counterpart for incommensurate systems and persist even outside the topological regime. The qualitative reason lies in the incomplete sampling of the Floquet zone in the case of rational drive and thus a possibility of preferential sampling of high Berry curvature regions.

\section{A. Technical interlude}

Before diving into the numerical results, several technical aspects of the simulation must be discussed. First, in our simulation, we integrated the Schrödinger equation to produce the unitary evolution operator $U(t)$. The Hamiltonian we use is a special case of Eq. (16):

$$
\begin{aligned}
\frac{\mathcal{H}}{\eta}= & \sin \left(\omega_{1} t+\phi_{1}\right) \sigma_{x}+\sin \left(\omega_{2} t+\phi_{2}\right) \sigma_{y} \\
& +\left[m-\cos \left(\omega_{1} t+\phi_{1}\right)-\cos \left(\omega_{2} t+\phi_{2}\right)\right] \sigma_{z},
\end{aligned}
$$

with the parameter $\eta$ defining the overall energy scale. Roughly speaking, the ratio of $\eta$ to the fastest frequency $\omega_{i}$ determines whether the strong or weak driving regime is realized. For the gap parameter $m$, we consider the topological $(|m|<2)$ and nontopological $(|m|>2)$ regimes. In our simulation, we constructed $U(t)$ as a product of the "infinitesimal" evolution matrices $\exp [-i \mathcal{H}(t) d t]$. Our time discretization step was $d t=0.001$, such that $\omega_{1} d t=10^{-4}$.

Given the unitary evolution operator, it is possible to calculate the integrated work done by each of the two drives. As discussed in Sec. IV B, the instantaneous power spent or absorbed by drive $i$ is 
$\frac{d W_{i}}{d t}=\left\langle\psi(t)\left|\frac{d h_{i}(t)}{d t}\right| \psi(t)\right\rangle=\left\langle\psi_{0}\left|U(t)^{\dagger} \frac{d h_{i}(t)}{d t} U(t)\right| \psi_{0}\right\rangle$.

[Here, $h_{i}(t)=\vec{h}_{i}(t) \cdot \vec{\sigma}$.] Therefore, we can define the work operator:

$$
\hat{W}_{i}=\int_{0}^{t} d t U(t)^{\dagger} \frac{d h_{i}(t)}{d t} U(t)
$$

such that $W_{i}=\left\langle\psi_{0}\left|\hat{W}_{i}\right| \psi_{0}\right\rangle$. Alongside $U(t)$, we also calculated the operators $\hat{W}_{i}$ for the two drives.

Finally, we must discuss the initial conditions for the simulation. We would like to have an initial state $\left|\psi_{0}\right\rangle$ that would maximize the energy transfer between the two drives in the topological case. For this purpose, we hark back to the analogy between the time-dependent Hamiltonian [e.g., as written in Eq. (28)] and the Hamiltonian of a real-space topological insulator. The analogy and the semiclassical logic of our topological pumping arguments suggest that a good choice for the initial state is an eigenstate of the instantaneous Hamiltonian at the beginning of the drive. When the drive is strong, $\eta \gg \omega_{1,2}$, the motion of the system in the photon-number space will be dictated by the semiclassical equations (23), with the Berry curvature determined by the band of the eigenstate $\left|\psi_{0}\right\rangle$. In this strong-drive limit, the eigenstates of the instantaneous initial Hamiltonian coincide with the Floquet eigenstates of a periodic system. For weaker drives, it might be advantageous to initialize the system into a Floquet state of a periodic system, which approximates the incommensurate two-drive system. We explore this briefly in Appendix E.

The fidelity of the initial state evolution, $\psi(t)=U(t) \psi_{0}$, with respect to the same-band instantaneous eigenstate of $\mathcal{H}(t), \psi_{i}(t)$, is crucial for effective driving in the incommensurate case. It is possible to write the fidelity as

$$
\begin{aligned}
\mathcal{F} & =\left\langle\psi(t)\left|P_{t}\right| \psi(t)\right\rangle \\
& =\operatorname{tr}\left[\left(\frac{1}{2}-\frac{\mathcal{H}(0)}{2 \epsilon(0)}\right) U^{\dagger}(t)\left(\frac{1}{2}-\frac{\mathcal{H}(t)}{2 \epsilon(t)}\right) U(t)\right] .
\end{aligned}
$$

From the definition of the projection operator at time $t, P_{t}$, it follows that

$$
\mathcal{F}=\frac{1}{2}\left[1+\langle\vec{\sigma}(t)\rangle \cdot \frac{\vec{h}(t)}{h(t)}\right] \equiv \cos ^{2} \frac{\theta(t)}{2},
$$

where $\theta(t)$ is the angle between the spin expectation and the instantaneous magnetic field at time $t$. If the spin is not in an instantaneous eigenstate, then the semiclassical equations of motion do not apply and the pumping effect is suppressed. The fidelity is robust, however, as long as the minimum gap in the band Hamiltonian significantly exceeds the drive frequencies,

$$
\Delta=\eta \min (|| m|-2|,|m|) \gg \omega_{1}, \omega_{2} .
$$

Indeed, one could consider the fidelity in the general framework of a repeated Landau-Zenner process. Every few cycles, the phases pass near the point(s) of the FloquetBrillouin zone where the gap reaches the minimum. In each passage, the probability for the spin to shift between the two bands is

$$
p \sim e^{-\Delta^{2} /(\partial H / \partial t)} \approx e^{-\left(\Delta^{2} / v|\vec{\omega}|\right)} .
$$

Therefore, the spin may maintain its fidelity for exponentially long times in the square of the strength parameter, $\eta$ :

$$
t_{\max } \sim \exp \left\{\frac{\eta^{2}[\min (|| m|-2|,|m|)]^{2}}{v|\vec{\omega}|}\right\} .
$$

When studying the rational frequency ratio case, it is natural to consider the Floquet eigenstates as initial states for the spin in addition to the instantaneous eigenstates. A rational frequency ratio implies a strict periodicity of the Hamiltonian. For $\omega_{1}=2 \pi / T p$ and $\omega_{2}=2 \pi / T q$, the Hamiltonian is periodic with period $\tau=T \operatorname{LCM}(p, q)$, with $\operatorname{LCM}(p, q)$ the lowest common multiple of $p$ and $q$. Typically, initialization into Floquet eigenstates results in faster pumping at short and intermediate times, but more importantly, since they are the eigenstates of $U(\tau)$, the pumping power itself is a periodic function of time.

\section{B. Incommensurate drives}

Let us demonstrate the pumping effects in the regime that best reflects the semiclassical limit, in which the Berry curvature effects are dominant. Simulating the system at $\eta=2$ yields near-perfect pumping effects essentially all the way to the topological transition at $m=2$. In Fig. 4, we see the energy transfer for several values of the gap parameter $m$, alongside the fidelity associated with the overlap of the initial state with the instantaneous eigenstate [as defined in Eq. (31)].

Throughout our analysis here, we use the incommensurate frequency pair $\omega_{1}=0.1$ and $\omega_{2}=\gamma \omega_{1}$, with $\gamma=$ $\frac{1}{2}(\sqrt{5}+1) \approx 1.618$, the golden ratio. The offset phases are $\phi_{1}=\pi / 10, \phi_{2}=0$. We explore the evolution up to times $t=10^{4}$, where the pumping effect is clearly visible. We vary $m$ and $\eta$ in our exploration.

The energy pumping rate indeed saturates to the one expected from the semiclassical analysis. For a system with Chern number 1 , we expect an average Berry curvature of $\Omega=(1 / 2 \pi)$ and an energy pumping rate of $d E / d t=$ $\left(\omega_{1} \omega_{2} / 2 \pi\right)=0.00257518$, as in Eq. (26). From linear regression of the plots in Fig. 4, we obtain excellent 


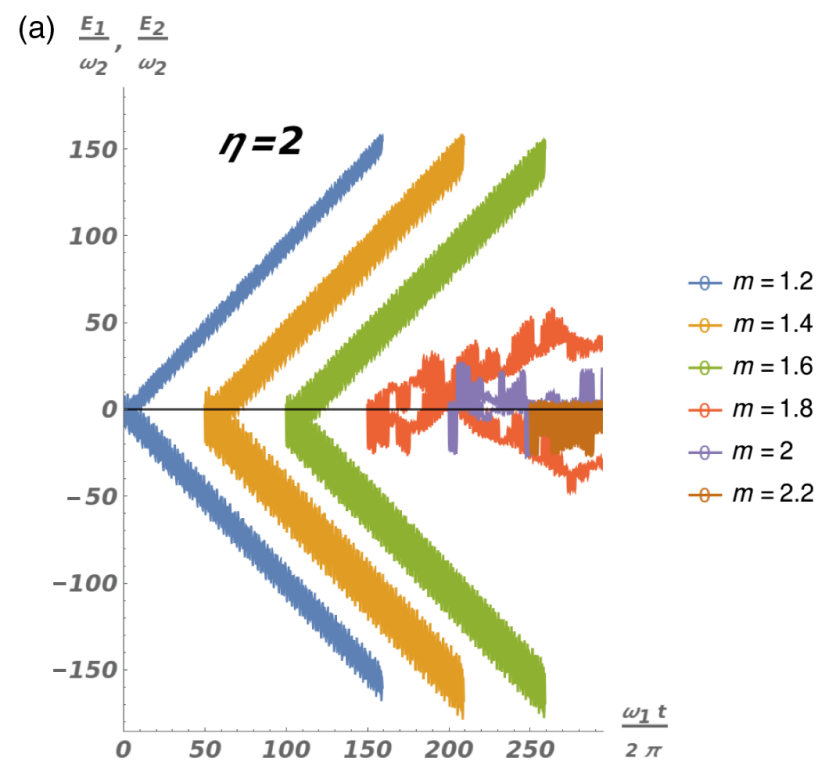

(b)

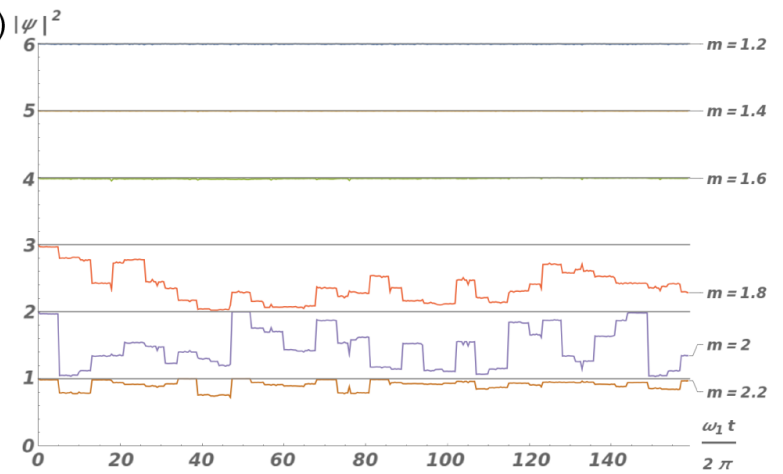

(c)

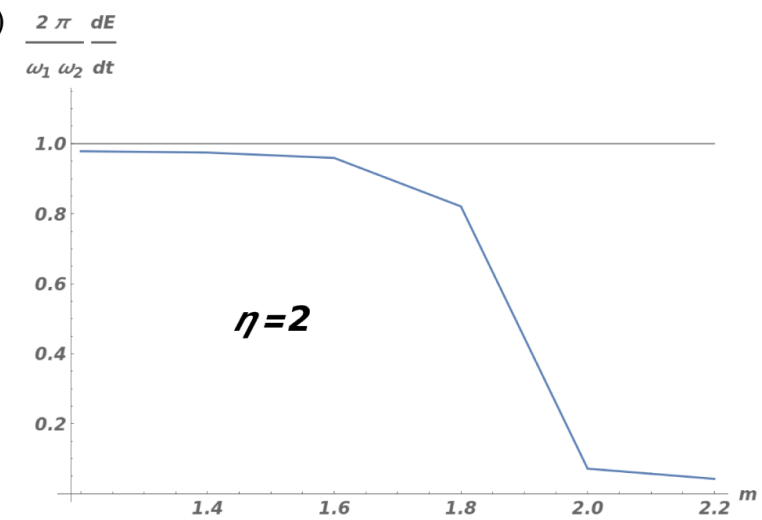

FIG. 4. The energy flow and fidelity for the model of Eq. (28) in the strong-drive regime, $\eta=2, \omega_{1}=0.1$, and $\omega_{2}=\gamma \omega_{1} \approx 0.1618$. (a) The total work done by drives 1 and 2 as a function of time for different $m$ 's. Each pair of lines is displaced on the time axis for clarity. The time axis is given in cycles of drive 1, while the work is normalized by $\omega_{2}$. The quantized rate corresponds to slope 1 . (b) Fidelity vs time of a state initialized in the instantaneous eigenstate of $\mathcal{H}(t=0)$ and measured against the eigenstate of $\mathcal{H}(t)$. All fidelities start at 1 at $t=0$ and are offset vertically for clarity. (c) The power pumped as a function of the parameter $m$ averaged up to time $t=2000$. The quantized pumping is marked by the gray line at $d E / d t=\omega_{1} \omega_{2} / 2 \pi$. The initial phases for these plots are $\phi_{1}=\pi / 10$, $\phi_{2}=0$. The strong-drive regime realizes the topological pumping prediction up to $m \sim 1.8$. Both fidelity and pumping deteriorate close to the phase transition because of the closing of the band gap.

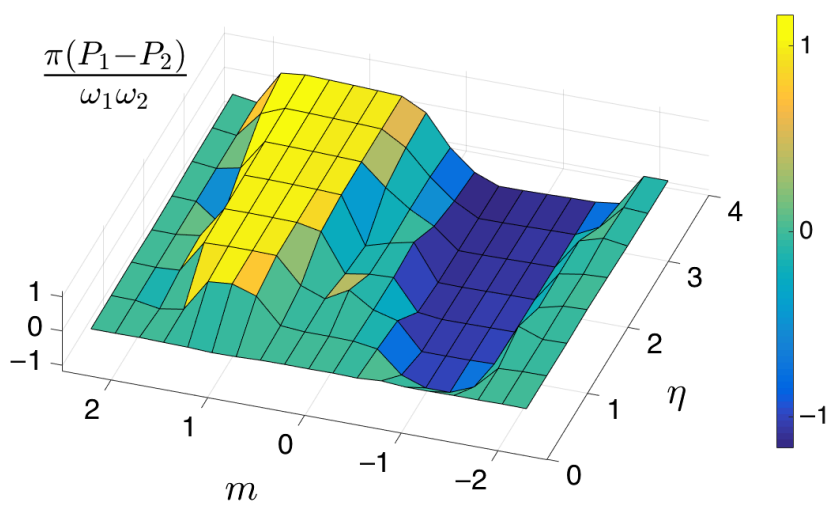

FIG. 5. Energy pumping between two frequency sources for varying $\eta$ and $m$. The parameters of the system for this plot are $\omega_{1}=0.1$ and $\omega_{2}=\gamma \omega_{1}$. The pumping rate was averaged over the time range $t<10000$. The theoretical (ideal) value for the chosen parameters is $\left|P_{1}-P_{2}\right|=\omega_{1} \omega_{2} / \pi \approx 0.00515036$.

agreement with the semiclassical theory. Similarly, the fidelity also remains near perfect throughout the evolution, up to $m \approx 1.8$. There, $\Delta=\eta|m-2|=0.4$, which is comparable to $\omega_{2} \approx 0.1618$, and the quasiadiabaticity condition is expected to break down. For larger values of $m$, outside the topological regime, the gap reopens and fidelity improves, but pumping becomes small.

As $\eta$ is reduced, the regime of ineffective pumping, where the gap $\Delta$ becomes comparable to or smaller than $\omega_{1,2}$, expands. This is visible in Fig. 5, which shows the pumping rate as a function of $m$ and $\eta$ for a broad region of parameter space. We explore smaller values of $\eta$, as well as the demise of this effect at weaker drives in Appendix F.

\section{Commensurate drive frequencies}

The energy pumping effect, and Eq. (22) in particular, can also be explored for a rational frequency ratio. In this case, however, it is not the Chern number that determines the effect but rather the integral of the Berry curvature along a closed path in the two-dimensional "Floquet zone," as shown in Fig. 3. We study this regime here for the frequency ratio $\omega_{1} / \omega_{2}=2 / 3$ (with $\omega_{1}=0.1$ ) and the strong drive $\eta=2$. Additional results for weaker drives are given in Appendix F.

As discussed in Sec. VA, when the frequency ratio is rational, the Hamiltonian is strictly periodic, and we can initiate the spin into a Floquet eigenstate of $\mathcal{H}(t)$. This results in a periodic pumping profile and typically larger pumping power, compared to the initialization into instantaneous eigenstates (a detailed comparison between the two initiation procedures is given in Appendix E).

The pumping profile for $1.2<m<2.2$, with offset phases $\phi_{1}=\pi / 10, \phi_{2}=0$, and Floquet eigenstate initialization is shown in Fig. 6. As can be seen, the energy pumping effect is present in the entire topological region. Indeed, the pumping rate may exceed the quantized 


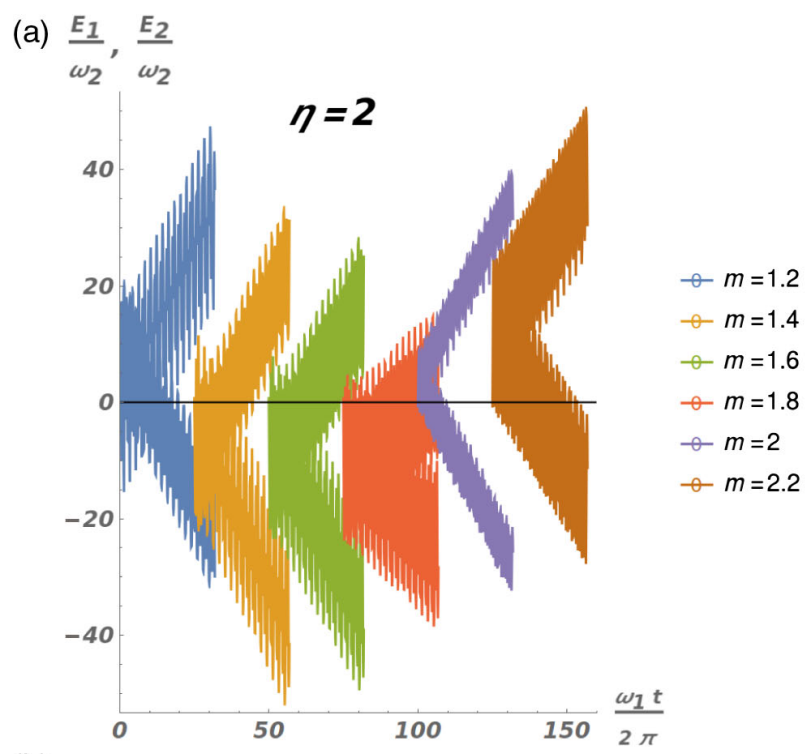

(b)

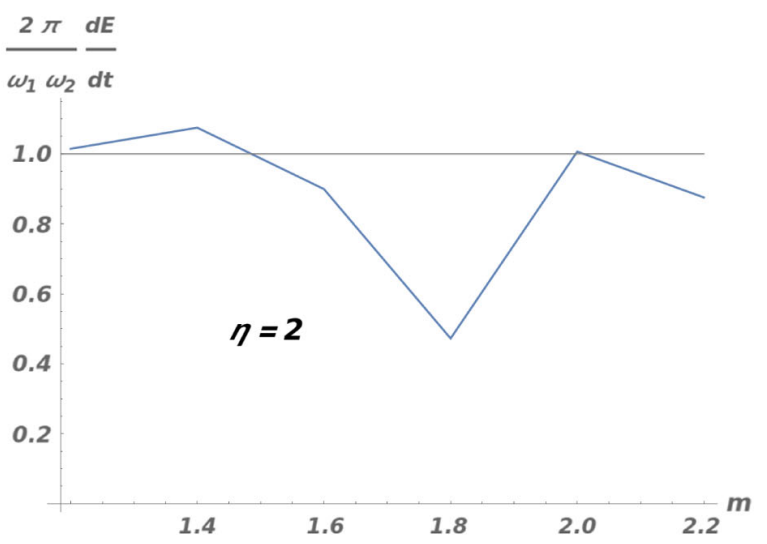

FIG. 6. The energy flow in the strong-drive regime, $\eta=2$, for rational frequency ratio, $\omega_{2}=(3 / 2) \omega_{1}$, with Floquet state initialization. (a) The total work done by drives 1 and 2 as a function of time for different $m$ 's. Each pair of lines is displaced on the time axis for clarity. (b) The power pumped as a function of the parameter $m$ averaged up to time $t=2000$. The pumping in the commensurate case could be stronger than its incommensurate counterpart. Furthermore, pumping may persist in the nontopological regime since the system does not average the Berry curvature of the entire Floquet zone. In the case of the offset angles chosen, even when $m=2.2$, the system explores regions with net positive Berry curvature. The gray line is at $d E / d t=\omega_{1} \omega_{2} / 2 \pi=0.002387$, signifying the pumping due to Berry curvature of the Chern number $C=1$ band. The initial phases for these plots are $\phi_{1}=\pi / 10, \phi_{2}=0$.

theoretical value since the energy pumping effect is now determined by the Berry curvature along a particular periodic path through the Floquet zone, rather than its average over the whole Floquet zone. Different paths can be selected by varying the offset phases. This dependence is illustrated in Fig. 7. It is also interesting to note that the offset-phase dependence is stronger for initialization into Floquet eigenstates, rather than into instantaneous eigenstates of the Hamiltonian (not shown). (a) $\underline{E_{1}}, \underline{E_{2}}$

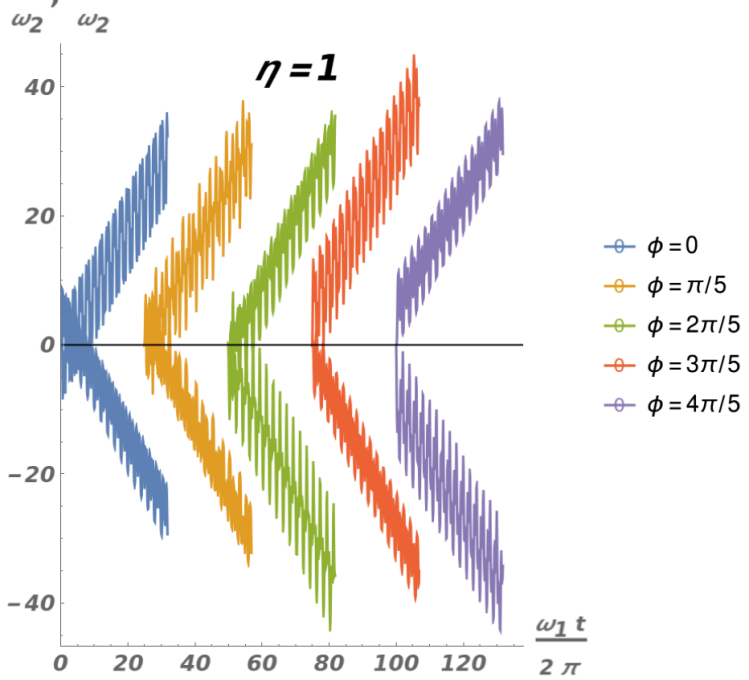

(b)

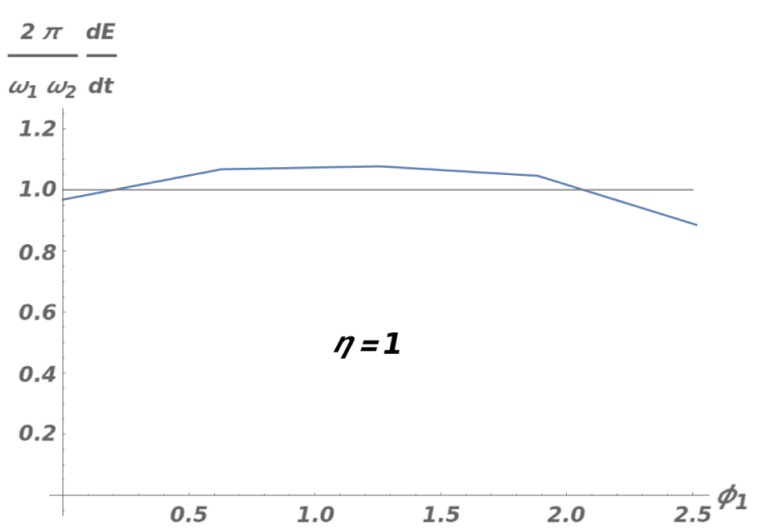

FIG. 7. Pumping profile for different offset phases for $\omega_{2}=(3 / 2) \omega_{1}$. (a) Accumulated work for Floquet initial states. (b) Average power for $t<2000$. We take $m=1, \eta=1$, and $\phi_{1}=$ $n \pi / 5$ with $n=0,1,2,3,4$. The Floquet zone path of this system for one offset phase combination is shown in Fig. 3. Floquet eigenstate initial conditions were used. For these parameters, there were no significant differences with instantaneous eigenstate initialization. Here, too, $\omega_{1} \omega_{2} / 2 \pi=0.002387$.

\section{TEMPORAL DISORDER AND THE RESTORATION OF PUMPING QUANTIZATION}

Phase noise of the drives leads to a surprising effect. Above, in Sec. V C, we emphasized and demonstrated that a commensurate pair of frequencies may induce pumping even in a parameter regime with a zero Chern number. In addition, we showed that even in the topological regime, pumping is not quantized, and it depends on the initial phase difference between the two mutually rational phases. Temporal disorder, however, is tantamount to averaging over all initial phases, and, in doing so, it restores the quantized nature of the pumping effects and makes it proportional to the Chern number of the underlying band structure. 
To demonstrate this principle, we consider a random noise in the drive's frequency. We choose the noise to be akin to

$$
\phi_{i}=\omega_{i} t+\phi_{i}^{(0)}+\delta_{i}(t)
$$

with $i=1,2$, and with $\overline{\dot{\delta}_{i}(t) \dot{\delta}_{i}\left(t^{\prime}\right)}=D_{i} \cdot(1 / \sqrt{\pi} \tau) e^{-\left(t-t^{\prime}\right)^{2} / \tau^{2}} \delta_{i j}$. Such disorder removes the periodicity of the commensurately driven problem. Also, whereas the commensurate drive explores only a discrete set of lines crossing the $\phi_{1}, \phi_{2}$ space, the noise makes the phases meander and explore the entire space. After time $T$, one expects a phase meander of order $\overline{\left(\Delta \phi_{i}\right)^{2}}=D_{i} T$.

Temporal disorder can also have detrimental effects. If the phase jitter is too strong, it will cause the fidelity of the spin state to drop [note that $\dot{\delta}_{i}(t)$ is an instantaneous correction to the frequency, and, if too large, spin cannot track the total magnetic field]. This can lead to a rapid deterioration of the pumping effect. However, by increasing the disorder correlation time, the fidelity can be preserved. Another effect is that if the angular frequencies of the drives fluctuate significantly, the pumping power would be affected. The quantized pumping corresponds to an integer number of photons of one frequency per cycle of the other. However, if the energy value of each photon varies with time, the pumping power also becomes noisy. Both of these effects are suppressed for large enough correlation time $\tau$-when the angular frequency fluctuations, $\overline{\dot{\delta}_{i}^{2}} \sim(D / \tau)$, are small $\sqrt{D / \tau} \ll \omega_{1,2}$.

Disorder thus causes the commensurate drive case to have the same energy pumping properties as the incommensurate case, in the long time limit, or for short times upon disorder averaging. A system with effective Chern number vanishing will not exhibit pumping, while a system in the topological regime will exhibit quantized pumping. Surprisingly, disorder reinforces the topological pumping quantization rule for any choice of drive frequencies.

To demonstrate the above conclusion, we simulated the commensurate case with varying degrees of disorder, disorder correlation time, and varying initial phases. We simulated over time spans corresponding to about 30 cycles of the slower drive and averaged over 20 disorder realizations. Figure 8 contrasts the pumping rate vs initial phase shift for a disorder-free system, and a system with $D_{1}=0$, $D_{2}=2.4 \times 10^{-3}$ (corresponding to a phase meandering of order $\Delta \phi_{2} \sim 2$ ) and correlation time $\tau=20$ for $\omega_{1}=0.1$, $\omega_{2}=\omega_{1} \cdot 3 / 2$. The simulations in the figure are all for $\eta=2$, with one example in the topological drive phase, $m=1.4$, and another in the trivial drive phase, $m=2.6$. We carry out a disorder average for the pumping rate, which is essentially equivalent to a long-time average in the adiabatic limit. The average is well quantized and independent of the initial phase, as expected.

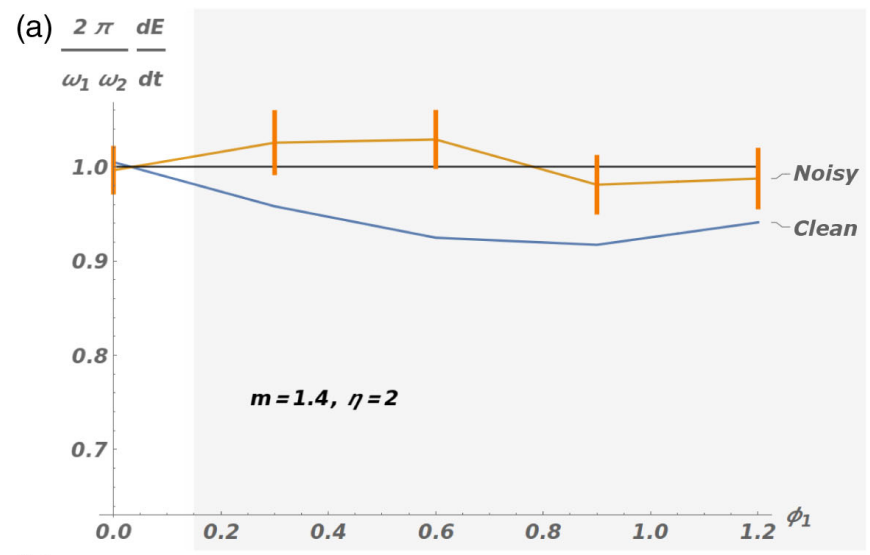

(b) $\frac{2 \pi}{\omega_{1} \omega_{2}} \frac{d E}{d t}$

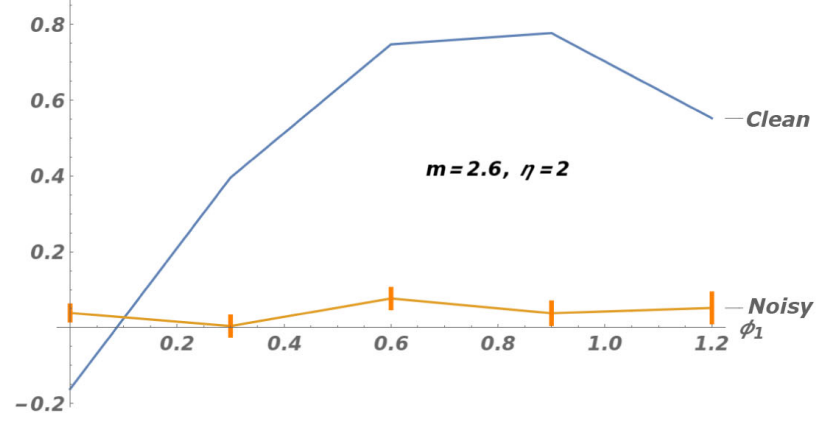

FIG. 8. A comparison of the energy pumping rates vs initial phase of drive $\omega_{1}, \phi_{1}$ (while holding $\phi_{2}=0$ ), for rational drive frequency ratios with and without temporal disorder. The results shown for the disordered cases are averaged over several realizations, and the disorder applied is a frequency random walk with temporal correlations as described in Sec. VI along with the additional parameters of the simulation. (a) The topological regime, with $m=1.4$. The disorder brings the average energy pumping rate back to the universal and initial-phaseindependent value of $\omega_{1} \cdot \omega_{2} / 2 \pi$. (b) The nontopological regime, with $m=2.6$. Although the rational frequency ratio allows for significant pumping well within the trivial driving phase, the effect lacks the topological protection, and disorder eliminates the pumping. The results in both plots are averaged over 20 realizations. The error bars represent the standard deviation of the average.

\section{DISCUSSION}

\section{A. Possible experimental realizations}

The two-frequency drive could be implemented in a variety of systems. The model we describe is that of a spin$1 / 2$ particle, subject to the magnetic field of two elliptically polarized light beams, normal to each other, in addition to a magnetic field normal to both beams. With the magnetic fields that correspond to intensities of $1 \mathrm{~W} / \mathrm{cm}^{2}$, and g-factor 2, the pumping effect could be realized with drives in frequencies of the order of $f \sim 1 \mathrm{MHz}$. One possibility is to have a gas of alkaline atoms (e.g., rubidium) trapped in an optical cavity. 
We discussed two types of initialization: into instantaneous eigenstates of the Hamiltonian and into the Floquet eigenstates (for the case of rational frequency ratios). In the limit of strong drive (slow frequency), they correspond to approximately the same state and similar pumping powers. We tacitly assumed that an arbitrary desired spin state can be created by the standard nuclear magnetic resonance (NMR)-like pulsing techniques and that the drive Hamiltonian can be turned on instantaneously. While theoretically consistent (the drive frequencies are slower than the frequency scales corresponding to the drive amplitudes), for an experiment, a simpler protocol would be desirable. One protocol to initialize into an instantaneous eigenstate is as follows.

(1) Apply a strong static field to the spin and wait a sufficiently long time for it to relax into its ground state. If desired, apply a $\pi$ pulse to put it into the excited state.

(2) Change the field slowly to the one that corresponds to the desired time-dependent Hamiltonian at time zero.

(3) Gradually increase drive frequencies from zero to the target values in the Hamiltonian.

An even simpler protocol can work as well, if the relaxation rate for the spin ( $T_{1}$ time) is shorter than the drive periods and the temperature is below the typical spin level splitting. One can simply start applying the stationary Hamiltonian, and spin itself will relax into a state closely approximating the instantaneous eigenstate. Indeed, our preliminary results on including dissipation show that pumping survives dissipation, and it can, in fact, help to stabilize the topological pumping.

The discussion in terms of electronic spins could be transported easily to other two-level systems. The quantum limit may be particularly amenable to experimental study using superconducting qubit systems [42]. The appeal of this scheme is both in the exquisite level of control over the qubit and in the extreme measurement sensitivity to the photon occupation numbers in the superconducting resonant circuitry. Yet another attractive feature of the superconducting devices, and Josephson junctions in particular, is the access to the ac Josephson effect in order to drive the superconducting phase by applying dc voltage. Manipulation of the superconducting phases has indeed been considered as a means to explore and control topological phases in multiterminal Josephson junctions $[43,44]$ and is mathematically analogous to the phase control provided by the ac drive that we consider in the present work.

Another interesting direction that one could explore is the use of electronic spin in systems with a strong spin-orbit coupling. For instance, once could consider a Weyl semimetal [45-48], with Hamiltonian near the Weyl nodes of

$$
H=v \vec{\sigma} \cdot(\vec{p}-e \vec{A})
$$

where $v$ is the Fermi velocity, $\vec{p}$ the electronic momentum, and $\vec{A}$ the vector potential of the light. We neglect Zeeman coupling altogether. Consider two beams that are elliptically polarized, propagating in the $x$ and $y$ directions, and affecting an electron in a state with $\vec{p}=p_{z} \hat{z}$. If the light's vector potential reaches amplitudes $e|\vec{A}|=e|\vec{E}| / \omega>p$, the electron will realize the Hamiltonian (16). The amplitudes realizable for such a scheme could be significantly higher, into the gigahertz regime. This assumes electric fields in excess of $100 \mathrm{~V} / \mathrm{m}$ and having access to electrons with momenta smaller than $h / 1 \mu \mathrm{m}$. Even larger fields would allow operation in even higher frequencies, possibly in the terahertz range. Similar arguments could be applied to the surface state of a topological insulator subject to a magnetic field.

Needless to say, realizations in a solid-state regime will have other issues such as disorder and the need for the electron to remain in a particular momentum state for many radiative cycles. Pursuing these directions will be the focus of future work.

\section{B. Generalizations}

There are two ways in which the model can be naturally generalized: by considering larger spins and by allowing the driving fields to have their own dynamics. The generalization of our results to larger spins is straightforward. Indeed, the dynamics of the expectation value of the spin is given by the Bloch equations, which are independent of the spin size [49],

$$
\dot{\vec{S}}(t)=i[H, \vec{S}(t)]=\vec{S}(t) \times \vec{h}(t) .
$$

The Bloch equations are linear in spin, and therefore, the precession frequency is independent of the spin size. The strong-drive criterion needed for the energy pumping for general spin size, in the language of the Bloch equations, corresponds to having the instantaneous Larmor frequency $|\vec{h}(t)|$ higher than the pump frequencies $\omega_{i}$-the same condition as for a spin- $1 / 2$ particle. On the other hand, from Eq. (22), the pumping power depends linearly on the spin size; thus, by taking a large magnetic particle with low magnetic anisotropy (e.g., a Yttrium iron garnet sphere [50]), or NMR or electron spin resonance systems [51], one can dramatically increase the pumping power to macroscopic levels.

One practical way to include the dynamics of the drive fields is by considering a closed system of spin and two electromagnetic cavities resonating at the drive frequencies. The cavities can be initialized into semiclassical coherent states to represent the periodic drives. The energy pumping will act to change both amplitudes (and phases) of the drives, eventually taking the system out of the topological regime. Our preliminary results indeed show that the 
system reaches the topological state boundary, after which the pumping direction reverses, albeit at a reduce rate.

Treating photons quantum mechanically raises several new questions. Perhaps most appealing is the possibility of using the two-frequency topological effects to pump energy from a laser of one frequency into a cavity with another frequency. Additional questions are as follows:

(i) What kinds of entangled spin-photon states are achievable?

(ii) Is there is a spontaneous transition into the pumping regime if one of the cavities is initialized into a vacuum state?

(iii) Can a superradiance transition occur in a system of multiple spins in the cavities? We leave these theoretical problems for future study.

\section{CONCLUSIONS}

In this work, we have demonstrated that it is possible to implement multidimensional topological band structures purely in the frequency space. The implementation relies on the ability to apply strong drives of incommensurate frequencies. The approach was illustrated using the twodimensional BHZ [30] model constructed by pumping a two-level system (spin-1/2 particle) with two elliptically polarized waves of incommensurate frequencies. The observable in this case is the quantized energy pumping power between the two drives, which is the direct analog of the quantized transverse Hall conductivity of the original real-space model. As such, the driving occurs in the topological regime of the model and remains effective as long as the drive's frequencies are lower than the minimal band gap in the model (quasiadiabatic regime).

The multiple-drive paradigm we propose here gives rise to the possibility of engineering emergent band models. These emergent band structures could produce interesting and potentially useful dynamics of photonic systems as well as access new entangled states of photons and matter. Furthermore, they are not limited to the two-drive example presented in our manuscript.

The implementation of the BHZ model in Floquet space that was our focus here is just one example of a model that can be implemented by means of strong ac driving. Many more exotic models can be built and analyzed by the same approach, by applying more drive frequencies (equivalent to going to higher-dimensional frequency spaces) or by pumping systems with more levels (including spatially extended ones). In particular, by applying three pump frequencies to a four-level system, one may be able to construct the 3D extension for the BHZ model [52]. The topological invariant in this case is the second Chern number that represents the magneto-electric response. Again, an effective electric field is built into the Floquet model by construction. It will be interesting to determine what observable the orbital magnetic response would correspond to in the Floquet implementation.
Pumping spatially extended systems by space-timedependent drives is another direction worth exploring. Mixing real space with Floquet space gives a simple interpretation of such classic effects as the Thouless pump in terms of the 2D quantum Hall effect (Appendix G) and can also help discover new phenomena. For instance, by pumping the 1D spatial lattice with two incommensurate frequencies, one may be able to construct 3D topological insulators with $S U(2)$ Landau levels [53,54]. By applying even more drives, one may be able to access and study even more exotic states, such as the eight-dimensional quantum Hall effect [55]. Finally, one can consider pumping nonlinear spatially quasiperiodic systems. By doing so, one may be able to access effective spatial dimensionalities higher than three. Indeed, in the context of perturbative nonlinear optics, 1D quasiperiodic spatial structures have been utilized to enable high-efficiency second and third harmonic generation $[56,57]$.

\section{ACKNOWLEDGMENTS}

The authors would like to thank J. Sau, M. Sanchez, A. Yacobi, V. Manucharyan, M. Gullans, M. Lukin, F. Nathan, and Y. Oreg for useful discussions. I. M. acknowledges support from the Department of Energy, Office of Basic Energy Science, Materials Science and Engineering Division. G. R. acknowledges support from the National Science Foundation (NSF) through Grant No. DMR1410435, as well as the Institute of Quantum Information and Matter, a NSF Frontier center funded in part by the Gordon and Betty Moore Foundation, and the Packard Foundation. B. H. acknowledges support from the STC Center for Integrated Quantum Materials, NSF Grant No. DMR-1231319. This research was supported in part by the National Science Foundation under Grant No. NSF PHY-1125915. We are also grateful to the Aspen Center for Physics, operating under NSF Grant No. 1066293, where part of the work was done.

\section{APPENDIX A: WEAK DRIVE}

When the drive is weak, the motion of the system in Floquet space is highly restricted. This can be seen from

$$
\left(E+\omega_{1} n+\omega_{2} m\right) \phi_{n m}^{\alpha}=h_{p q}^{\alpha \beta} \phi_{n-p, m-q}^{\beta} .
$$

Because of the large energy mismatches between neighboring sites, the "particle" can hop on the lattice only along a 1D "Manhattan" path that skirts the constant-energy line, e.g., $E+\omega_{1} n+\omega_{2} m=0$. The sites of the corresponding 1D model have on-site energies given by

$$
u_{n m}=\omega_{1} n+\omega_{2} m=\sqrt{\omega_{1}^{2}+\omega_{2}^{2}}(n \sin \alpha+m \cos \alpha) .
$$

This on-site energy is proportional to the distance between a lattice point $(n, m)$ and the line cutting through the lattice at 
angle $\alpha$. For $\tan \alpha$ irrational, this distance is a quasiperiodic quantity; hence, the system represents a 1D lattice with quasiperiodic on-site potential. This can be compared to the well-studied case of the Fibonacci quasicrystal [58]. In that case, $\tan \alpha=\gamma$ and the on-site energies are all the same, but the hoppings are different if they derive from the "vertical" ( $m$-direction) or the "horizontal" ( $n$-direction) hopping. Clearly, we can also introduce such asymmetry by choosing $h_{01} \neq h_{01}$, however, the mapping to $1 \mathrm{D}$ only applies if $\omega_{i} \gg|h|$, and thus, it is not possible to exactly replicate the limit of pure bond "disorder." The spectrum of this problem is dense everywhere because of the symmetry $E \rightarrow E+$ $\omega_{1} n+\omega_{2} m$ accompanied by wave-function translation. Because of the quasiperiodic potential, we expect the wave functions to be localized along the effective 1D lattice.

\section{APPENDIX B: COMMENSURATE DRIVE FREQUENCIES: DETAILED TREATMENT}

When drives are incommensurate, the wave function $\phi_{\vec{n}}$ in Eq. (11) is defined on the whole 2D Floquet plane since all frequencies $n \omega_{1}+m \omega_{2}$ are unique. Suppose now that $\omega_{1} / \omega_{2}=p / q$, with $p<q$ mutually prime integers. It is easy to see that if $p=1$, then $n \omega_{1}$ exhausts all the possible frequencies. For $p \neq 1$, the number of independent frequencies is $p$ times larger. Thus, in the commensurate case, Floquet space is "compactified" into a strip of width $p$ along the $\omega_{1}$ axis. The boundary conditions are periodic along the $\omega_{2}$ direction, but with an "offset" of $q$, introducing a shear (twist).

Let us see how this comes about in detail. As before, the Schrödinger equation is

$$
i \partial_{t} \psi^{\alpha}(t)=H^{\alpha \beta}(t) \psi^{\beta}(t),
$$

with $H(t)=\mathcal{H}\left(\omega_{1} t, \omega_{2} t\right)$, where $\mathcal{H}\left(\theta_{1}, \theta_{2}\right)$ is $2 \pi$ periodic in $\theta_{1,2}$. Hence,

$$
\mathcal{H}\left(\omega_{1} t, \omega_{2} t\right)=\sum_{\vec{m}} h_{\vec{m}} e^{-i \vec{\omega} \cdot \vec{m} t}=\sum_{\text {strip }} \tilde{h}_{\vec{m}} e^{-i \vec{\omega} \cdot \vec{m} t},
$$

where we combined all terms with the same frequencies, thus reducing summation to a strip of frequencies described above.

Similarly, the Floquet representation for the wave function is

$$
\begin{aligned}
\psi(t) & =e^{-i E t} \phi(t)=\sum_{\vec{n}} e^{-i E t-i \vec{\omega} \cdot \vec{n} t} \phi_{\vec{n}} \\
& =\sum_{\text {strip }} e^{-i E t-i \vec{\omega} \cdot \vec{n} t} \tilde{\phi}_{\vec{n}} .
\end{aligned}
$$

Substitution into the Schrödinger equation leads to the expected result: When the action of the Hamiltonian seems to take the system outside the strip, a shift by $\pm(-q, p)$ brings it back into the strip. This corresponds to the "sheared" periodic boundary conditions in the strip.

An alternative selection of unique frequencies is a tilted ribbon with the regular (nonsheared) periodic boundary conditions. The construction is the same as in the case of carbon nanotubes with helicity $(p, q)$. The Hamiltonian induces hopping on the nanotube; the energy-conserving dynamics corresponds to hopping along the nanotube circumference (perimeter $\sqrt{p^{2}+q^{2}}$ ).

Note that if $p, q \gg 1$, then the compactification should not be noticeable, and one does not expect any difference between irrationally and such rationally related frequencies (e.g., if one constructs topological insulators in Floquet space).

\section{APPENDIX C: FLOQUET EIGENSTATES IN QUASIPERIODIC POTENTIALS}

When drives are quasiperiodic (irrational frequency ratio), the Floquet theorem does not directly apply since one cannot define the evolution operator over the full period. Nonetheless, a quasiperiodic drive with irrational $\omega_{2} / \omega_{1}=\gamma$ can be approximated by a periodic one, $\omega_{2} / \omega_{1}=p / q$ ( $p$ and $q$ are positive, relatively prime integers). For instance, for $\gamma$ the golden mean, $q$ and $p$ can be chosen as consecutive Fibonacci numbers. Then, the drive is periodic, with the period $T=p T_{1}=q T_{2}$, and the Floquet theorem can be applied. Hence, one can ask whether in the limit of $p, q \rightarrow \infty$, the FE converge to unique states. These could be naturally defined as the Floquet eigenstates for the case of incommensurate drives.

For a periodic drive, FE have the form $\Psi(t)=e^{-i E t} \Phi(t)$, with $\Phi(t)$ periodic. The FE are the eigenstates of the evolution operator over the period

$$
U(T)=\mathcal{T} \exp -i \int_{0}^{T} H\left(t^{\prime}\right) d t^{\prime} .
$$

For a two-level system (TLS), this is a $2 \times 2$ matrix that can be easily computed and diagonalized to find FE.

\section{Observations}

To test the convergence of Floquet eigenstates, we studied Hamiltonian Eq. (28) with parameters $\eta=1$ and $m=1.5$. We considered $\omega_{2} / \omega_{1}=\lim _{i \rightarrow \infty} p_{i} / q_{i}=r$, with irrational $r$ chosen as $\gamma$ (with $\omega_{1}=0.3$ ) and $\sqrt{2}$ (with $\omega_{1}=0.5$ ). We used continued fraction representations of $\gamma=[1 /(1+\{1 /[1+(1 / 1+\cdots)]\})]$. and $\sqrt{2}=$ $1+[1 /(2+\{1 /[2+(1 / 2+\cdots)]\})]$ terminated at a finite level to obtain the sequences of (the best [59]) rational approximations. The largest-denominator approximations that we considered were $2584 / 1597$ for $\gamma$ and $3363 / 2378$ for $\sqrt{2}$. In Fig. 9, the deviation, $|\Delta \Psi|_{i}=\left|\Psi_{p_{i} / q_{i}}-\Psi_{p_{\max } / q_{\max }}\right|$, is plotted as a function of $\left|p_{i} / q_{i}-r\right|$ for the two cases [the reference values are $\Psi_{2584 / 1597} \approx(0.094+0.496 i, 0.863)^{\dagger}$ 


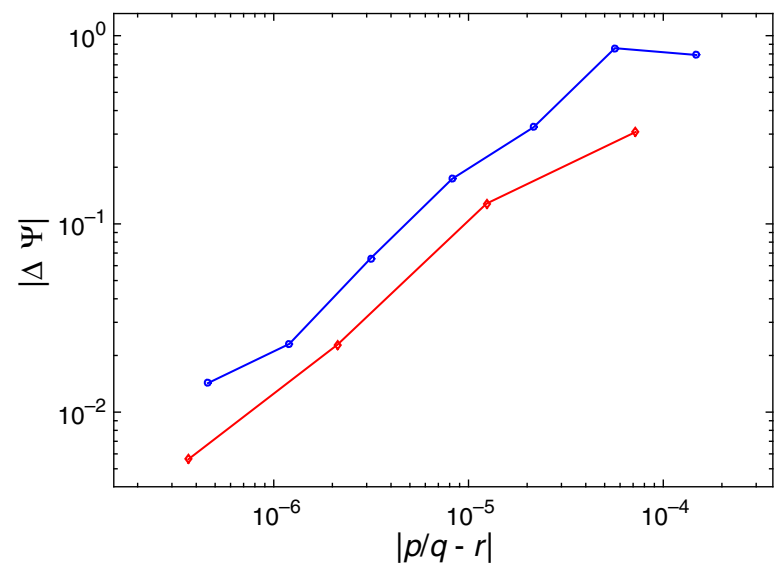

FIG. 9. Convergence of Floquet eigenfunctions for $\omega_{1} / \omega_{2}=$ $p_{i} / q_{i} \rightarrow r$, where $r$ is irrational [the golden mean, $\gamma=1.6180 \ldots$ (blue line) and $\sqrt{2}$ (orange line)]. Note that $|\Delta \Psi|$ is calculated relative to the best rational approximation used $(2584 / 1597$ for $\gamma$ and 3363/2378 for $\sqrt{2}$ ); it is approximately linear in $|p / q-r|$. Parameters of the Hamiltonian are described in the text.

and $\left.\Psi_{3363 / 2378} \approx(-0.230-0.446 i, 0.865)^{\dagger}\right]$. Both show approximately linear convergence, indicating the existence of the Floquet eigenstates for incommensurate drive frequencies. We also find, as expected, that in the limit of small frequencies $\left(\omega_{i} \ll \eta\right)$, the FE are nearly the eigenstates of the instantaneous Hamiltonian.

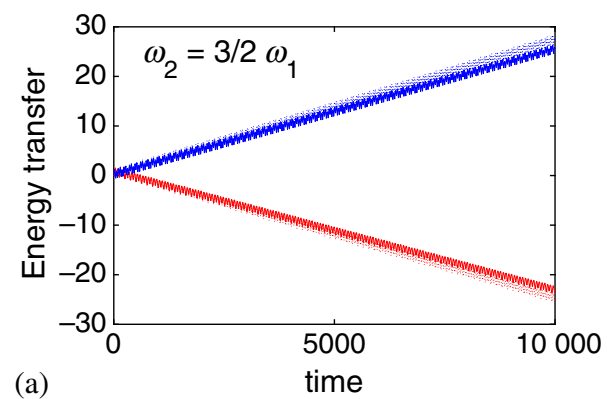

(a)

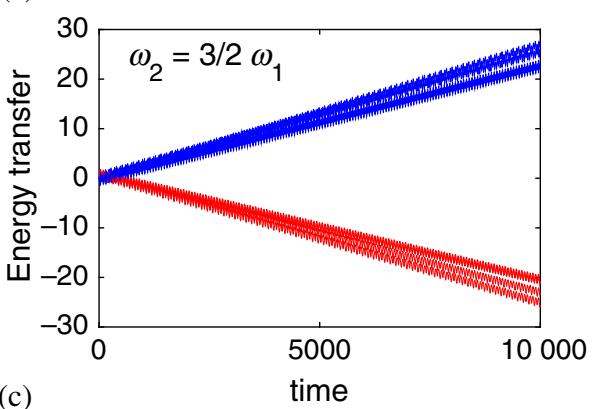

Note that we only tested the FE convergence in the gapped phase. While we have not tested it explicitly, we expect that in the gapless points $(|m|=0,2)$, the limit may not exist since small changes in frequencies would lead to topologically distinct trajectories in the Floquet zone.

\section{APPENDIX D: QUANTUM PUMP ANALOGY}

It is tempting to rewrite the Berry curvature of Eq. (25) as

$$
\Omega_{\vec{q}}=\hat{z} i \frac{1}{2 \epsilon_{\vec{q}}^{2}} \operatorname{tr}\left(P_{\vec{q}}\left[\frac{\partial \mathcal{H}_{\vec{q}}}{\partial q_{1}}, \frac{\partial H_{\vec{q}}}{\partial q_{2}}\right]\right)
$$

[this is easy to see since $\left.\operatorname{tr}\left[P_{\vec{q}} \mathcal{H}_{\vec{q}}\left(\partial P_{\vec{q}} / \partial q_{i}\right)\right]=0\right]$. This result leads to a formula for the pump power,

$$
\frac{1}{2} \frac{\partial\left(E_{1}-E_{2}\right)}{\partial t}=\frac{\omega_{1} \omega_{2}}{2 \epsilon_{\vec{q}}^{2}} \operatorname{tr}\left(P_{\vec{q}}\left[\frac{\partial \mathcal{H}_{\vec{q}}}{\partial q_{1}}, \frac{\partial H_{\vec{q}}}{\partial q_{2}}\right]\right) .
$$

Equation (D2) bears close similarity to the quantum pumping formula [60], except that instead of the $S$ matrix, the derivatives in the commutator are of a Hamiltonian. Indeed, the photons that enter and leave the driven system do not have a conservation law (since they have different frequencies), and therefore, they cannot be assigned an $S$ matrix. This equation may suggest that a generalization of the quantum pumping formula for conserved but nonquantized quantities, such as energy, may exist.
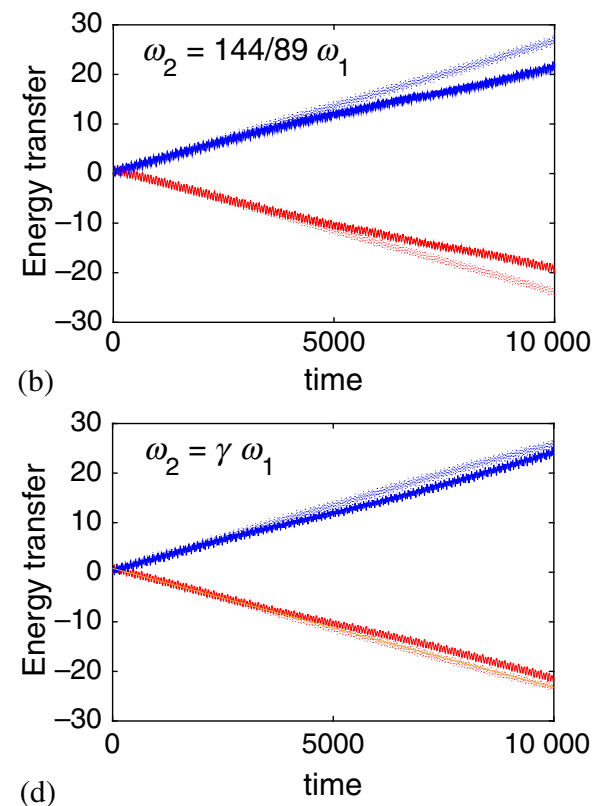

FIG. 10. Comparison of evolution starting from instantaneous (solid lines) and Floquet (dotted lines) eigenstates. Rational frequencies in panels (a)-(c) are approximations of the golden mean $\gamma$. The Hamiltonian scale is $\eta=1$ and $\omega_{1}=0.1$, with $\omega_{2}$ stated on the plots. Panel (c) illustrates how the energy transfer for the rational drive depends on the relative phase, $\phi_{2}-\phi_{1}=0,1.5,3,4.5$, starting from the respective instantaneous eigenstates. The variation is due to sampling of different closed lines in the phase Brillouin zone. Panel (d) illustrates how initializing into a Floquet eigenstate (computed for $\omega_{2}=144 / 89 \omega_{1}$ ) enhances pumping at long times for the irrational drive. 

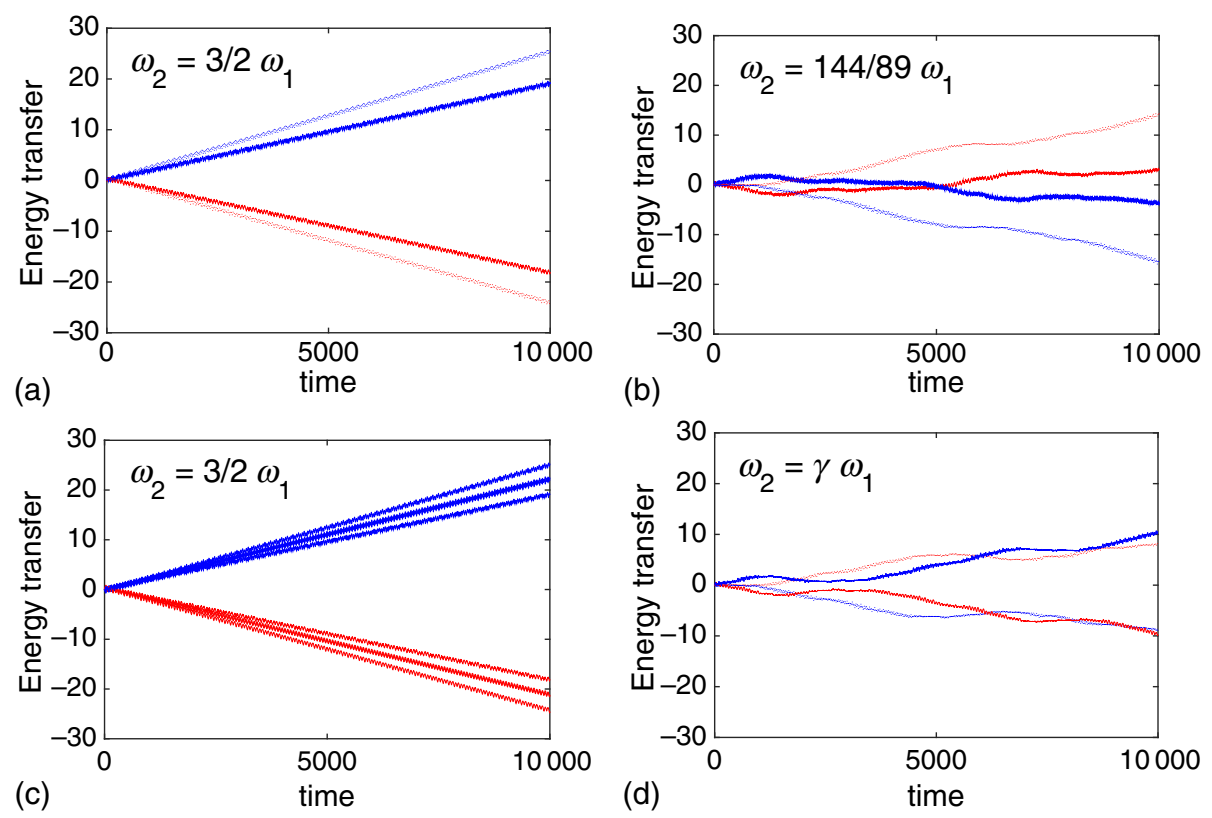

FIG. 11. Same as Fig. 10, but for $\eta=0.5$.

\section{APPENDIX E: FLOQUET VS INSTANTANEOUS EIGENSTATE INITIALIZATION}

In Fig. 10, we consider two rational approximations to the golden mean: $\omega_{2}=3 / 2 \omega_{1}$ and $\omega_{2}=144 / 89 \omega_{1}$, for $\eta=1$ and $\omega_{1}=0.1$. First, even in the case of small commensuration, $\omega_{2} / \omega_{1}=2 / 3$, there is significant pumping. The rate of pumping is similar to the one for a nearby incommensurate ratio. As mentioned in Sec. IV E, for the commensurate drives, the Floquet zone is not fully sampled, and hence, the results can depend on the relative phase between the drives. Indeed, varying the initial phase shift does lead to different pumping rates [see Fig. 10(c)].

In Figs. 10(a), 10(b), and 10(d), we have compared the initializations in the instantaneous eigenstates (solid lines) and the Floquet eigenstates (dotted lines). In all cases we considered, we found that initialization in the Floquet eigenstates leads to faster pumping. For incommensurate drive frequencies, Fig. 10(d), the initialization was in the Floquet eigenstate of $\omega_{2}=144 / 89 \omega_{1}$ (approximation to the incommensurate FE discussed in Appendix C). That initialization led to faster and more linear pumping, with
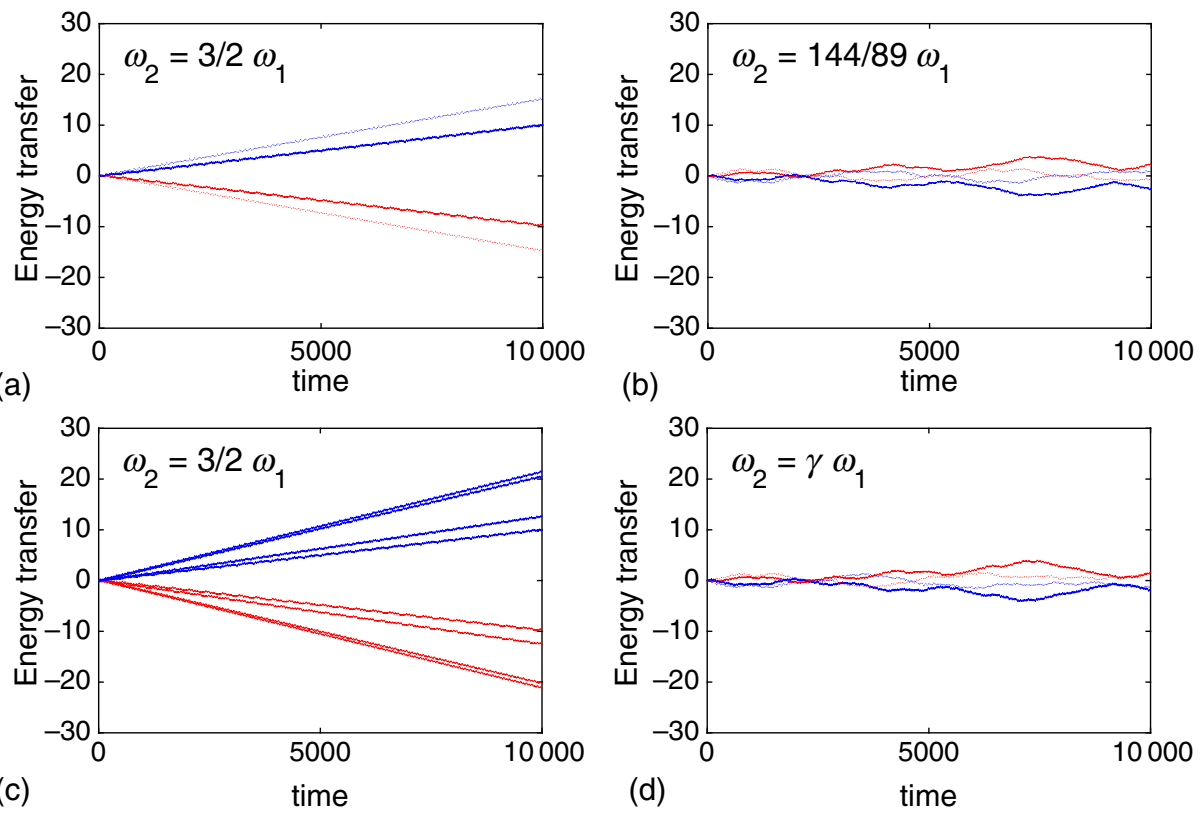

FIG. 12. Same as Fig. 10, but for $\eta=0.2$. 
the value near the theoretical upper limit, Eq. (26). The reason for faster pumping can be traced to higher average fidelity in the FE.

In Figs. 11 and 12, we consider the energy pumping, for the same parameters as in Fig. 10, but for reduced Hamiltonian scales, $\eta=0.5$ and $\eta=0.2$, respectively. Consistently with the expectation, the energy pumping is suppressed, particularly strongly for larger denominator (and irrational) fractions $\omega_{1} / \omega_{2}$. Interestingly, for small denominators, e.g., $2 / 3$, the pumping persists even for relatively small $\eta$.

\section{APPENDIX F: ADDITIONAL NUMERICAL RESULTS}

In addition to the numerical results we presented in the text, we explored the intermediate and weak-drive regimes of the double-drive BHZ model. We present these numerical results here for completeness. Unless stated otherwise, $\omega_{1}=0.1$ and $\omega_{2}=\gamma \omega_{1}$, where $\gamma$ is the golden mean.

\section{Intermediate drives, $\boldsymbol{\eta}=0.5,1$}

For $\eta$ values that exceed $\omega_{1,2}$, but not by much, we still see a strong pumping effect deep in the topological regime. The effect subsides, however, well before the phase boundary between the topological and trivial parameter regimes [Figs. 13(a) and 14(a)]. As can be seen from Figs. 13(b) and 14(b), this is a result of the fidelity being lost after a finite time in parameter ranges close to or beyond the topological transition into the trivial range. This is associated with the system exploring parts of the Floquet zone where the band gap of the underlying BHZ model is comparable to the drive frequencies.

Figures 13(c) and 14(c) show the power absorbed by the drives averaged over the first 2000 time units of the evolution as a function of the gap parameter $m$ for $\eta=0.5,1$. The transition between the pumping regime and the trivial regime is quite abrupt.

\section{Weak drives, $\eta \leq \omega_{1,2}$}

To explore the weak-drive regime, we considered $\eta=0.1$. As Fig. 15 suggests, initializing the system with the instantaneous eigenstate of the Hamiltonian $\mathcal{H}(0)$ results in negligible pumping. Initialization in a Floquet eigenstate of a periodic approximation does not qualitatively change the result, as can be seen from Fig. 12(d).

\section{APPENDIX G: THOULESS PUMP, RELOADED}

In this section, we provide the reinterpretation of the Thouless pump [61] using the Floquet lattice construction. The Thouless pump is a classic example of a driven system that shows quantized transport behavior. Consider a one-dimensional tight-binding lattice, $x_{i}=i$, with a spatially and time-dependent on-site potential, (a) $E_{30}, E_{2}$

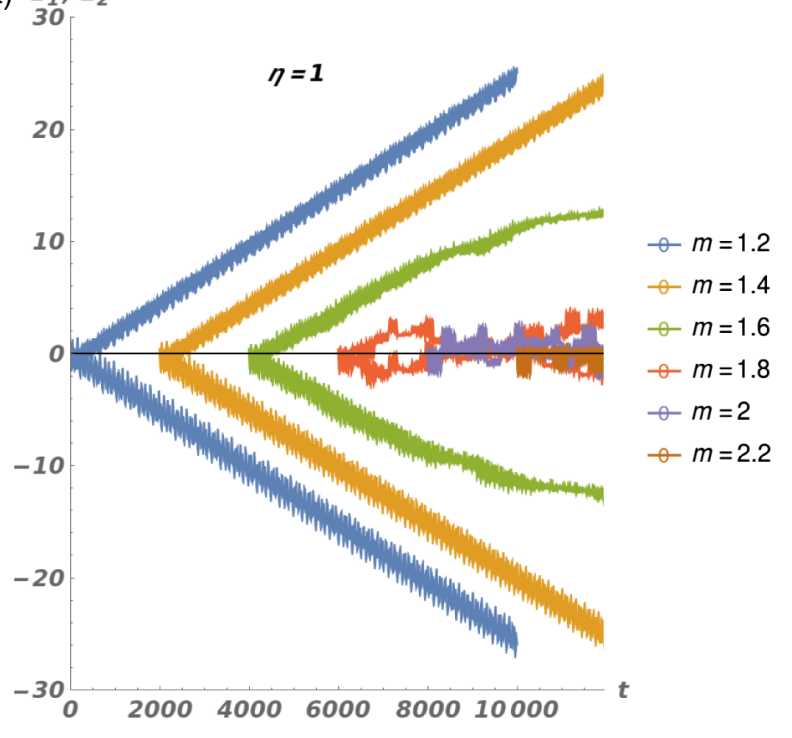

(b)
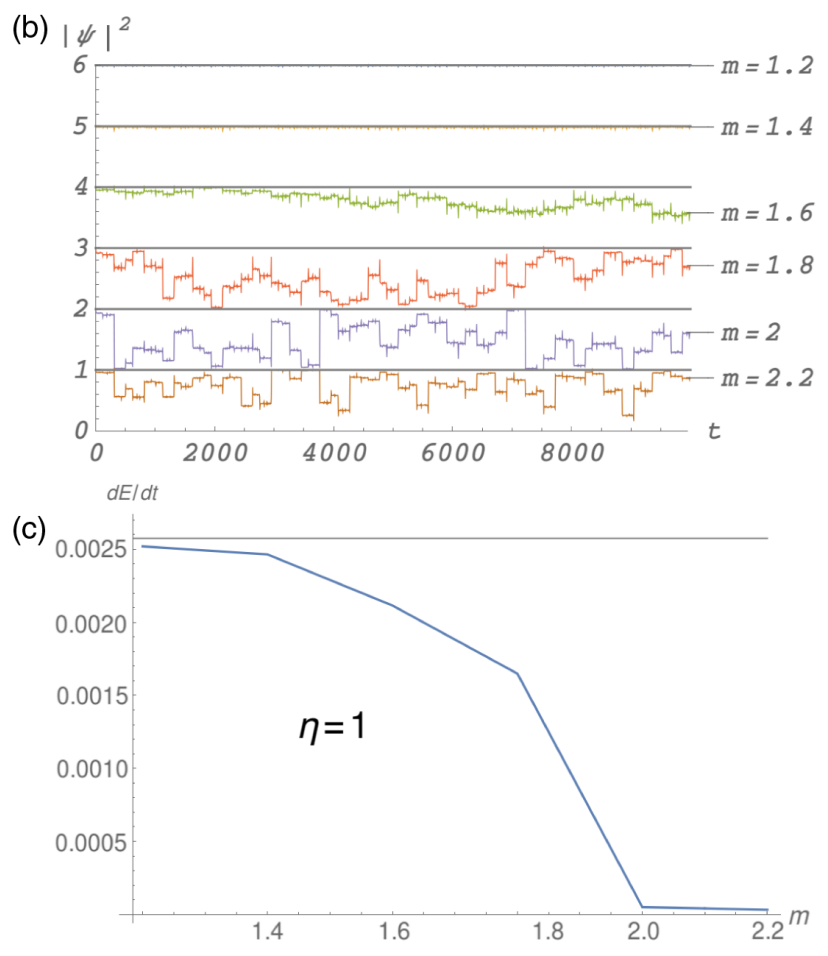

FIG. 13. Work (a) and fidelity (b) in the intermediate drive regime for $\eta=1$. We initialized the system with the instantaneous eigenstate of $\mathcal{H}(0)$, where the initial phases for these plots are $\phi_{1}=\pi / 10, \phi_{2}=0$. (c) Power pumped averaged for $t<2000$ as a function of gap parameter $m$.

$V(x, t)=A \cos \left(\omega_{0} t-k x_{i}\right)$. The potential is time periodic, and thus, we can take advantage of the Floquet transformation described in Sec. III. As a result, we obtain a two-dimensional lattice-one spatial dimension that no longer has spatially varying potential, and one frequency dimension, with the superimposed linear potential corresponding to an applied electric field of strength $\omega_{0}$. The hopping in the positive (negative) frequency direction is 

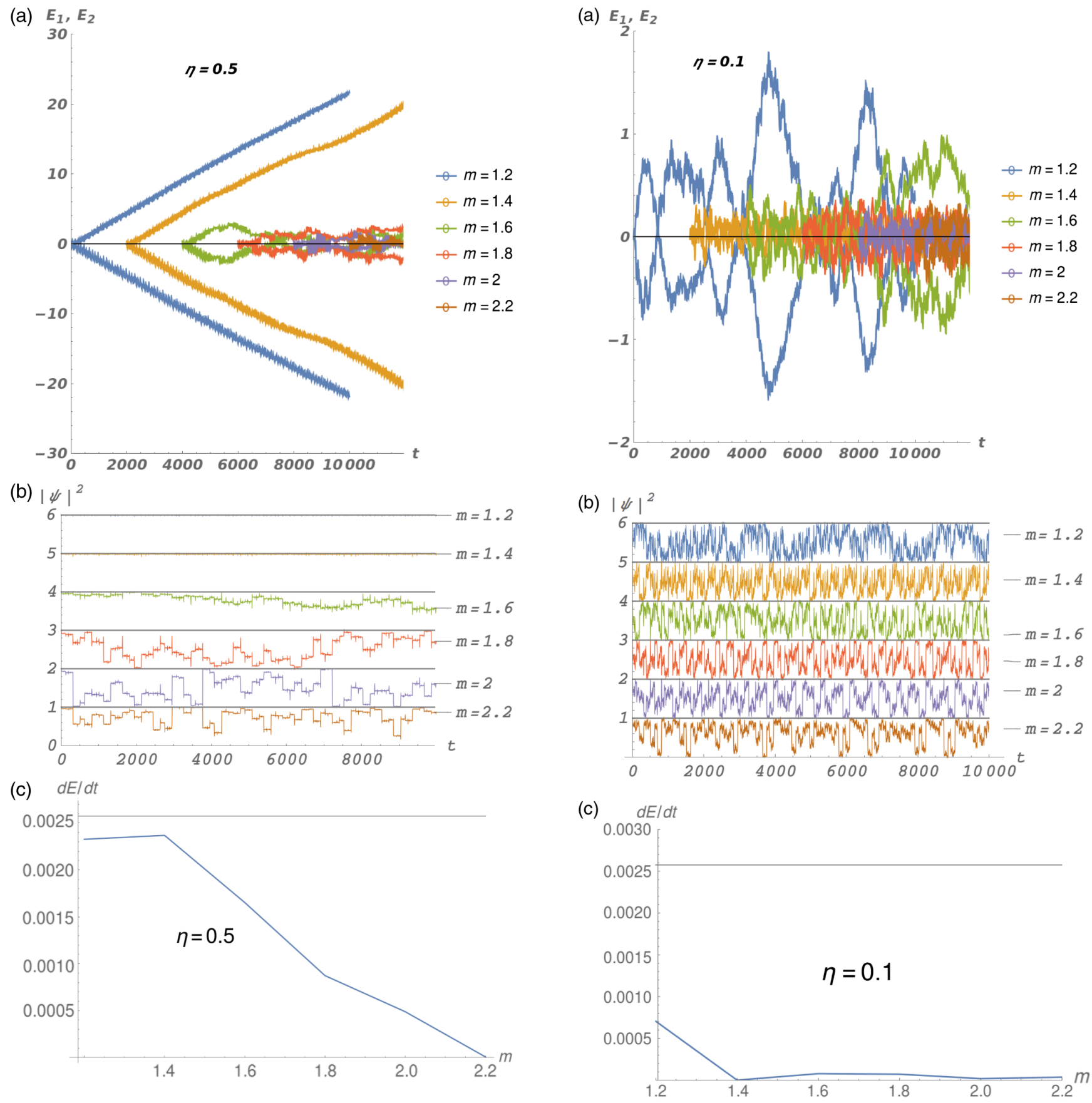

FIG. 14. Work (a) and fidelity (b) in the intermediate drive regime for $\eta=0.5$. We initialized the system with the instantaneous eigenstate of $\mathcal{H}(0)$, where the initial phases for these plots are $\phi_{1}=\pi / 10, \phi_{2}=0$. (c) Power pumped averaged for $t<2000$ as a function of gap parameter $m$.

$A e^{ \pm i k x_{i}}$. The phase factor corresponds to an effective linearin- $x$ vector potential pointing in the $y$ (frequency) direction. This is equivalent to a uniform magnetic field of strength $k$ piercing the lattice. We therefore see that the Thouless pump in 1D maps onto a quantum Hall problem in 2D. Indeed, the topological invariant in Ref. [61] is nothing but the Chern number in the mixed coordinates of spatial momentum and time momentum (phase). Because of the

FIG. 15. Work and fidelity in the weak-drive regime. (a) Energy transfers for $\eta=0.1$. (b) Fidelities of the initial state, an instantaneous eigenstate of $\mathcal{H}(0)$, and the instantaneous eigenstates of $\mathcal{H}(t)$. The initial phases for these plots are $\phi_{1}=\pi / 10, \phi_{2}=0$. (c) Power pump averaged for $t<2000$ as a function of gap parameter $m$.

presence of crossed electric and magnetic fields, a particle placed in the lattice will experience drift, with velocity orthogonal to both, of the magnitude given by their ratio, $v_{\text {drift }}=\omega_{0} / k$. This is nothing but the speed of the potential in the original problem, which yields the pumping result of the Thouless pump. 
[1] T. Oka and H. Aoki, Photovoltaic Hall Effect in Graphene, Phys. Rev. B 79, 081406 (2009).

[2] J.-i. Inoue and A. Tanaka, Photoinduced Transition between Conventional and Topological Insulators in TwoDimensional Electronic Systems, Phys. Rev. Lett. 105, 017401 (2010).

[3] N. H. Lindner, G. Refael, and V. Galitski, Floquet Topological Insulator in Semiconductor Quantum Wells, Nat. Phys. 7, 490 (2011).

[4] N. H. Lindner, D. L. Bergman, G. Refael, and V. Galitski, Topological Floquet Spectrum in Three Dimensions via a Two-Photon Resonance, Phys. Rev. B 87, 235131 (2013).

[5] T. Kitagawa, T. Oka, A. Brataas, L. Fu, and E. Demler, Transport Properties of Nonequilibrium Systems under the Application of Light: Photoinduced Quantum Hall Insulators without Landau Levels, Phys. Rev. B 84, 235108 (2011).

[6] M. S. Rudner, N. H. Lindner, E. Berg, and M. Levin, Anomalous Edge States and the Bulk-Edge Correspondence for Periodically Driven Two-Dimensional Systems, Phys. Rev. X 3, 031005 (2013).

[7] P. Titum, E. Berg, M. S. Rudner, G. Refael, and N. H. Lindner, Anomalous Floquet-Anderson Insulator as a Nonadiabatic Quantized Charge Pump, Phys. Rev. X 6, 021013 (2016).

[8] N. H. Lindner, G. Refael, and V. Galitski, Floquet Topological Insulator in Semiconductor Quantum Wells, Nat. Phys. 7, 490 (2011).

[9] F. Nathan and M. S. Rudner, Topological Singularities and the General Classification of Floquet-Bloch Systems, New J. Phys. 17, 125014 (2015).

[10] R. Roy and F. Harper, Periodic Table for Floquet Topological Insulators, arXiv:1603.06944 [Phys. Rev. X (to be published)].

[11] R. Roy and F. Harper, Floquet Topological Phases with Symmetry in All Dimensions, Phys. Rev. B 95, 195128 (2017).

[12] C. W. von Keyserlingk and S. L. Sondhi, Phase Structure of One-Dimensional Interacting Floquet Systems. I. Abelian Symmetry-Protected Topological Phases, Phys. Rev. B 93, 245145 (2016).

[13] A. C. Potter, T. Morimoto, and A. Vishwanath, Classification of Interacting Topological Floquet Phases in One Dimension, Phys. Rev. X 6, 041001 (2016).

[14] C. W. von Keyserlingk and S. L. Sondhi, ID Many-Body Localized Floquet Systems II: Symmetry-Broken Phases, Phys. Rev. B 93, 245146 (2016).

[15] C. W. von Keyserlingk, V. Khemani, and S. L. Sondhi, Absolute Stability and Spatiotemporal Long-Range Order in Floquet Systems, Phys. Rev. B 94, 085112 (2016).

[16] D. V. Else, B. Bauer, and C. Nayak, Prethermal Time Crystals and Floquet Topological Phases without Disorder, Phys. Rev. X 7, 011026 (2017).

[17] I.-D. Potirniche, A. C. Potter, M. Schleier-Smith, A. Vishwanath, and N. Y. Yao, Floquet Symmetry-Protected Topological Phases in Cold Atomic Systems, arXiv: 1610.07611 [Phys. Rev. Lett. (to be published)].

[18] D. Abanin, W. De Roeck, and F. Huveneers, A Theory of Many-Body Localization in Periodically Driven Systems, Ann. Phys. (Amsterdam) 372, 1 (2016).
[19] A. Lazarides, A. Das, and R. Moessner, Fate of Many-Body Localization Under Periodic Driving, Phys. Rev. Lett. 115, 030402 (2015).

[20] L. Zhang, V. Khemani, and D. A. Huse, A Floquet Model for the Many-Body Localization Transition, Phys. Rev. B 94, 224202 (2016).

[21] Y. H. Wang, H. Steinberg, P. Jarillo-Herrero, and N. Gedik, Observation of Floquet-Bloch States on the Surface of a Topological Insulator, Science 342, 453 (2013).

[22] M. C. Rechtsman, J. M. Zeuner, Y. Plotnik, Y. Lumer, D. Podolsky, F. Dreisow, S. Nolte, M. Segev, and A. Szameit, Photonic Floquet Topological Insulators, Nature 496, 196 (2013).

[23] J. Zhang, P. W. Hess, A. Kyprianidis, P. Becker, A. Lee, J. Smith, G. Pagano, I.-D. Potirniche, A. C. Potter, A. Vishwanath, N. Y. Yao, and C. Monroe, Observation of a Discrete Time Crystal, arXiv:1609.08684.

[24] L. Yuan, Y. Shu, and S. Fan, Photonic Gauge Potential in a System with a synthetic Frequency Dimension, Opt. Lett. 41, 741 (2016).

[25] Q. Lin, M. Xiao, L. Yuan, and S. Fan, Photonic Weyl Point in a Two-Dimensional Resonator Lattice with a Synthetic Frequency Dimension, Nat. Commun. 7, 13731 (2016).

[26] T. Ozawa, H. M. Price, N. Goldman, O. Zilberberg, and I. Carusotto, Synthetic Dimensions in Integrated Photonics: From Optical Isolation to Four-Dimensional Quantum Hall Physics, Phys. Rev. A 93, 043827 (2016).

[27] P. A. Kalugin, A. Yu. Kitaev, and L. S. Levitov, Al(86)Mn(14): A Six-Dimensional Crystal, JETP Lett. 41, 145 (1985).

[28] D. Levine and P. J. Steinhardt, Quasicrystals-A New Class of Ordered Structures, Phys. Rev. Lett. 53, 2477 (1984).

[29] D. Shechtman, I. Blech, D. Gratias, and J. W. Cahn, Metallic Phase with Long-Range Orientational Order and No Translational Symmetry, Phys. Rev. Lett. 53, 1951 (1984).

[30] B. A. Bernevig, T. L. Hughes, and S.-C. Zhang, Quantum Spin Hall Effect and Topological Phase Transition in HgTe Quantum Wells, Science 314, 1757 (2006).

[31] S. Mukamel, Principles of Nonlinear Optical Spectroscopy (Oxford University Press on Demand, New York, 1999), Vol. 6.

[32] G. Floquet, On Linear Differential Equation with Periodic Coefficients, in Annales Scientifiques de l'École Normale Supérieure (1883), Vol. 12, pp. 47-88.

[33] F. Bloch, Über die Quantenmechanik der Elektronen in Kristallgittern, Z. Phys. 52, 555 (1929).

[34] A. Di Carlo, P. Vogl, and W. Pötz, Theory of Zener Tunneling and Wannier-Stark States in Semiconductors, Phys. Rev. B 50, 8358 (1994).

[35] L. Yuan and S. Fan, Optica 3, 1014 (2016).

[36] T.-S. Ho, S.-I. Chu, and J. V. Tietz, Semiclassical ManyMode Floquet Theory, Chem. Phys. Lett. 96, 464 (1983).

[37] G. Casati, I. Guarneri, and D. L. Shepelyansky, Anderson Transition in a One-Dimensional System with Three Incommensurate Frequencies, Phys. Rev. Lett. 62, 345 (1989).

[38] D. Xiao, M.-C. Chang, and Q. Niu, Berry Phase Effects on Electronic Properties, Rev. Mod. Phys. 82, 1959 (2010).

[39] It is important here that $\omega_{1} / \omega_{2}$ is irrational; for the rational case, see below. 
[40] T. Nakanishi, T. Ohtsuki, and M. Saitoh, Stark Ladders in a Two-Dimensional Tight-Binding Lattice, J. Phys. Soc. Japan 62, 2773 (1993).

[41] G. Sundaram and Q. Niu, Wave-Packet Dynamics in Slowly Perturbed Crystals: Gradient Corrections and Berry-Phase Effects, Phys. Rev. B 59, 14915 (1999).

[42] V. E. Manucharyan, J. Koch, L. I. Glazman, and M. H. Devoret, Fluxonium: Single Cooper-Pair Circuit Free of Charge Offsets, Science 326, 113 (2009).

[43] B. van Heck, S. Mi, and A. R. Akhmerov, Single Fermion Manipulation via Superconducting Phase Differences in Multiterminal Josephson Junctions, Phys. Rev. B 90, 155450 (2014).

[44] R.-P. Riwar, M. Houzet, J. S Meyer, and Y. V. Nazarov, Multi-terminal Josephson Junctions as Topological Matter, Nat. Commun. 7, 11167 (2016).

[45] X. Wan, A. M. Turner, A. Vishwanath, and S. Y. Savrasov, Topological Semimetal and Fermi-Arc Surface States in the Electronic Structure of Pyrochlore Iridates, Phys. Rev. B 83, 205101 (2011).

[46] A. A. Burkov and L. Balents, Weyl Semimetal in a Topological Insulator Multilayer, Phys. Rev. Lett. 107, 127205 (2011).

[47] A. A. Burkov, M. D. Hook, and L. Balents, Topological Nodal Semimetals, Phys. Rev. B 84, 235126 (2011).

[48] B. Singh, A. Sharma, H. Lin, M. Z. Hasan, R. Prasad, and A. Bansil, Topological Electronic Structure and Weyl Semimetal in the TlBiSe ${ }_{2}$ Class of Semiconductors, Phys. Rev. B 86, 115208 (2012).

[49] Anatole Abragam, The Principles of Nuclear Magnetism (Oxford University Press, New York, 1961), Vol. 32.
[50] E. G. Spencer, R. C. LeCraw, and A. M. Clogston, Low-Temperature Line-Width Maximum in Yttrium Iron Garnet, Phys. Rev. Lett. 3, 32 (1959).

[51] A. M. Tyryshkin, S. A. Lyon, A. V. Astashkin, and A. M. Raitsimring, Electron Spin Relaxation Times of Phosphorus Donors in Silicon, Phys. Rev. B 68, 193207 (2003).

[52] L. Fu, C. L. Kane, and E. J. Mele, Topological Insulators in Three Dimensions, Phys. Rev. Lett. 98, 106803 (2007).

[53] Y. Li and C. Wu, High-Dimensional Topological Insulators with Quaternionic Analytic Landau Levels, Phys. Rev. Lett. 110, 216802 (2013).

[54] Y. Li, S.-C. Zhang, and C. Wu, Topological Insulators with SU(2) Landau Levels, Phys. Rev. Lett. 111, 186803 (2013).

[55] B. A. Bernevig, J. Hu, N. Toumbas, and S.-C. Zhang, EightDimensional Quantum Hall Effect and "Octonions", Phys. Rev. Lett. 91, 236803 (2003).

[56] S.-n. Zhu, Y.-y. Zhu, and N.-b. Ming, Quasi-Phase-Matched Third-Harmonic Generation in a Quasi-Periodic Optical Superlattice, Science 278, 843 (1997).

[57] K. Fradkin-Kashi, A. Arie, P. Urenski, and G. Rosenman, Multiple Nonlinear Optical Interactions with Arbitrary Wave Vector Differences, Phys. Rev. Lett. 88, 023903 (2001).

[58] C. Janot, Quasicrystals (Springer, New York, 1994).

[59] A. Hurwitz, Ueber die Angenäherte Darstellung der Irrationalzahlen Durch Rationale Brüche, Math. Ann. 39, 279 (1891).

[60] P.W. Brouwer, Scattering Approach to Parametric Pumping, Phys. Rev. B 58, R10135 (1998).

[61] D. J. Thouless, Quantization of Particle Transport, Phys. Rev. B 27, 6083 (1983). 